\title{
Supporting Information: Multiphase Photochemistry of Iron-Chloride \\ Containing Particles as a Source of Aqueous Chlorine Radicals and Its Effect on Sulfate Production
}

\author{
Masao Gen ${ }^{1,2}$, Ruifeng Zhang ${ }^{1}$, Yongjie $\mathrm{Li}^{3}$, Chak K. Chan ${ }^{1 *}$
}

${ }^{1}$ School of Energy and Environment, City University of Hong Kong, Tat Chee Avenue,

Kowloon, Hong Kong, China

${ }^{2}$ Faculty of Frontier Engineering, Institute of Science and Engineering, Kanazawa University,

Kakuma-machi, Kanazawa 920-1192, Japan

${ }^{3}$ Department of Civil and Environmental Engineering, Faculty of Science and Technology,

University of Macau, Macau 999078, China

\section{Corresponding Author}

*Author to whom correspondence should be addressed

Email: chak.k.chan@cityu.edu.hk

Telephone: +(852)-3442-5593

This PDF file includes:

Pages S1 to S25

Tables S1 to S4

Figures S1 to S21

Text S1 to S6 


\section{Text S1. Calculations of photon fluxes received by sample particles}

A chemical actinometer of 2-nitrobenzaldehyde $(2 \mathrm{NB})^{1}$ was used to quantify photon fluxes at 280-420 nm (Figure S2), received by a sample particle in the photochemical flow cell. 2NB was dissolved in methanol, and the resulting solution was atomized. The atomized droplets were deposited on a hydrophobic substrate (Model 5793, YSI Inc.). The decay of 2NB in the droplet under UV-vis irradiation was measured by Raman spectrometer (EnSpectr R532, EnSpectr) (Figure S19). Following earlier work, ${ }^{1}$ the $2 \mathrm{NB}$ photo-decay rate constant, $j(2 \mathrm{NB})$, was estimated:

$\ln \left(\frac{[2 \mathrm{NB}]_{t}}{[2 \mathrm{NB}]_{0}}\right)=-j(2 \mathrm{NB}) t$

where $[2 \mathrm{NB}]_{\mathrm{t}}$ and $[2 \mathrm{NB}]_{0}$ are the concentrations of $2 \mathrm{NB}$ at a given time and time zero, respectively. $j(2 \mathrm{NB})$ obtained was then used to calculate the photon fluxes using the following equation:

$j(2 \mathrm{NB})=\frac{2.303 \times 10^{3} \mathrm{~cm}^{3} \mathrm{~L}^{-1}}{N_{\mathrm{A}}} \times \sum\left(I_{\lambda} \times \Delta \lambda \times \varepsilon_{2 \mathrm{NB}, \lambda} \times \phi_{2 \mathrm{NB}, \lambda}\right)$

where $N_{\mathrm{A}}$ is Avogadro's number; $I_{\lambda}$ and $\Delta \lambda$ are the actinic flux (photon $\mathrm{cm}^{-2} \mathrm{~s}^{-1} \mathrm{~nm}^{-1}$ ) and the wavelength interval between data points (nm), respectively; $\varepsilon_{2 \mathrm{NB}, \lambda}$ and $\Phi_{2 \mathrm{NB}, \lambda}$ are the base-10 molar absorptivity $\left(\mathrm{M}^{-1} \mathrm{~cm}^{-1}\right)$ and quantum yield $\left(\right.$ molecule photon $\left.{ }^{-1}\right)$ of $2 \mathrm{NB}$, respectively. The summation is over all irradiation wavelengths $\lambda$. $\varepsilon_{2 \mathrm{NB}, \lambda}$ was experimentally determined using UV-vis spectrometer (UV-3600, SHIMADZU) (Figure S20) and $\Phi_{2 \mathrm{NB}, \lambda}$ of 0.41 was found to be constant at $\lambda$ between 280 and $405 \mathrm{~nm}^{1}$ and applied for all $\lambda$.

\section{Text S2. Kinetic modeling of sulfate production}

Sulfate production was reproduced by numerically solving a series of differential equations for each chemical species. Table S2 lists all reactions incorporated in the model. As discussed in the main text, the photolysis rate constants for iron chlorides (i.e., $\mathrm{FeCl}^{2+}$ and $\left.\mathrm{FeCl}_{2}{ }^{+}\right)(\mathrm{R} 1$ and R2 in Table S2) were set as fitting parameters and were varied to match the modelled sulfate production with the observation. The fitted parameters are shown in Table S3. In this study, $\mathrm{Fe}^{2+}$ is oxidized into $\mathrm{Fe}^{3+}$ via the reactions of $\mathrm{R} 33-36$, and $\mathrm{R} 40$. The rate constants of $\mathrm{R} 35$ and R36 have not been reported and hence were determined as fitting parameters using the experiment 1 (Table S3). The fitted values of the constants in R35 and R36 were obtained to be $8.0 \times 10^{5} \mathrm{M}^{-1} \mathrm{~s}^{-1}$ and $5.65 \times 10^{-2} \mathrm{~s}^{-1}$, respectively, and were applied to the all the rest of the experiments. When nitrate was also present in the system, nitrate photolysis was incorporated in the model (R41 and R42). Its photolysis rate constants were also set as the fitting parameters and found to be $(3.8-9.0) \times 10^{-7} \mathrm{~s}^{-1}$, which is similar to the reported value $\left(1.23 \times 10^{-7} \mathrm{~s}^{-1}\right)$ found in the bulk nitrate solution under atmospherically relevant irradiation. ${ }^{2}$ Furthermore, nitrate is known to reacts with $\mathrm{Fe}^{2+}$ for the regeneration of $\mathrm{Fe}^{3+}$ (R40). ${ }^{3,4}$ However, to the best of our knowledge, its reaction rate constant has not been reported. Therefore, this rate constant was set as the fitting parameter in the experiment 2 and determined to be $1.52 \times 10^{-2}\left(\mathrm{M}^{-2} \mathrm{~s}^{-1}\right)$. The determined rate constant was applied to all the rest of the experiments.

In the presence of oxalic acid or malonic acid as an organic ligand, the organic compounds bind with $\mathrm{Fe}^{3+}$ for the complexation. The iron-organic complexes are subjected to photolysis (R52 and R62-64 for oxalic acid and malonic acid, respectively). Similar to photolysis of iron chlorides (R1 and R2), the photolysis rate constants of the iron-organic complexes were also set as fitting parameters and determined to be $(1.7-2.9) \times 10^{-2}$ and $(0.42-2.5) \times 10^{-4} \mathrm{~s}^{-1}$ for R52 and R62-64, respectively. The photolysis rate constant of Fe(III)oxalate is found to be two orders of magnitude higher than that of $\mathrm{Fe}(\mathrm{III})$-malonate, which is consistent with the difference of the quantum yields between Fe(III)-oxalate (0.88) and Fe(III)- 
malonate (0.0022) for $\mathrm{Fe}(\mathrm{II})$ formation. The mechanisms of the photolysis of $\mathrm{Fe}(\mathrm{III})$-malonate were adopted from the literature (Figure S21). ${ }^{5}$

Text S3. Calculations of reactive uptake coefficients of $\mathrm{SO}_{2}$ into sulfate

Following earlier work, ${ }^{6}$ we calculated reactive uptake coefficient of $\mathrm{SO}_{2}$ into sulfate, $\gamma_{\mathrm{SO} 2}$, using the experimentally determined sulfate production rate, $\mathrm{d}\left[\mathrm{SO}_{4}{ }^{2-}\right] / \mathrm{dt}$ :

$\gamma_{\mathrm{SO} 2}=\frac{d\left[\mathrm{SO}_{4}^{2-}\right]}{d t} / Z$

$Z=\frac{1}{4} \omega_{\mathrm{SO}_{2}} A_{\mathrm{S}}\left[\mathrm{SO}_{2}\right]$

$A_{\mathrm{S}}=4 \pi r_{\mathrm{p}}^{2} \times\left(4 \pi r_{\mathrm{p}}^{3} / 3\right)^{-1}$

where $\omega_{\mathrm{SO} 2}$ and $\left[\mathrm{SO}_{2}\right]$ are the thermal velocity of and the concentration of $\mathrm{SO}_{2}$ vapor, respectively; $A_{\mathrm{S}}$ and $r_{\mathrm{p}}$ are the specific surface area of a particle of interest and the particle radius. As shown in Figure 2 in the main text, $\mathrm{d}\left[\mathrm{SO}_{4}{ }^{2-}\right] / \mathrm{dt}$ decreases with time and hence the initial $\mathrm{d}\left[\mathrm{SO}_{4}{ }^{2-}\right] / \mathrm{dt}$ in an approximately linear fashion was used to calculate $\gamma_{\mathrm{SO} 2}$.

\section{Text S4. Comparison of sulfate production pathways}

In this study, sulfate is produced via the five pathways: the chain reactions initiated by the reactions of $\mathrm{SO}_{3}{ }^{-}$and chlorine radicals ( $\mathrm{R} 15, \mathrm{R} 16$, and $\mathrm{R} 21$, thereafter chlorine pathway), the reaction of $\mathrm{Fe}^{2+}$ and $\mathrm{SO}_{4}^{-}$( $\mathrm{R} 33, \mathrm{SO}_{4}^{-}$pathway), oxidation by $\mathrm{H}_{2} \mathrm{O}_{2}\left(\mathrm{R} 39, \mathrm{H}_{2} \mathrm{O}_{2}\right.$ pathway), oxidation by $\mathrm{N}(\mathrm{III})$ (R44, N(III) pathway), and oxidation by $\mathrm{NO}_{2}\left(\mathrm{R} 45, \mathrm{NO}_{2}\right.$ pathway) in the presence of nitrate. Figures S15 and S16 show the comparisons of sulfate production rates from the pathways. The results show that the chlorine pathway contributes to $>96 \%$ of total sulfate production and is found to be the major pathway in this study. Note that when the $\mathrm{Fe}^{3+}$ concentrations are reduced to below millimolar levels, the contribution of the chlorine pathway becomes significantly low, whereas the $\mathrm{N}(\mathrm{III})$ and $\mathrm{NO}_{2}$ pathways are dominant $(>94 \%$ of total sulfate production).

\section{Text S5. ${ }^{\circ} \mathrm{OH}$ and $\mathrm{Cl}^{-}$reaction as a potential source of $\mathrm{Cl}^{\bullet}$}

The reaction of $\mathrm{Cl}^{-}$and $\mathrm{OH}$ indirectly produces $\mathrm{Cl}^{-}$via the intermediate formation of $\mathrm{ClOH}^{-}$ and the $\mathrm{Cl}^{\circ}$ production rate is sensitive to $\mathrm{pH}{ }^{7}$

$\mathrm{Cl}^{-}+{ }^{\cdot} \mathrm{OH} \rightarrow \mathrm{ClOH}^{-}$

$\mathrm{ClOH}^{-} \rightarrow \mathrm{Cl}^{-}+{ }^{\cdot} \mathrm{OH}$

$\mathrm{ClOH}^{-}+\mathrm{H}^{+} \rightarrow \mathrm{H}_{2} \mathrm{O}+\mathrm{Cl}^{\cdot} \quad \mathrm{R} 2$

The reaction firstly produces $\mathrm{ClOH}^{-}$that subsequently undergoes two reactions: (R1) regeneration of $\mathrm{Cl}^{-}$and $\mathrm{OH}$, and (R2) its reaction with $\mathrm{H}^{+}$to produce $\mathrm{Cl}^{\circ}$. Using reported rate constants of $6.1 \times 10^{9} \mathrm{~s}^{-1}$ and $2.1 \times 10^{10} \mathrm{M}^{-1} \mathrm{~s}^{-1}$ in the literature, ${ }^{7}$ the reaction rate of (R1) is higher than that of $(\mathrm{R} 2)$ at $\mathrm{pH}>0$. Hence, under the atmospherically relevant $\mathrm{pH}$ range between 0 and 6, we assume that the $\mathrm{Cl}^{\circ}$ production from the reaction of $\mathrm{Cl}^{-}$and ${ }^{\circ} \mathrm{OH}$ through (R2) may be insignificant. 


\section{Text S6. Estimation of reactivity of glyoxal with chlorine radicals}

Oxidation of glyoxal by chlorine radicals was performed to demonstrate their oxidation capacity for organic compounds. Particles containing $\mathrm{Fe}^{3+}$, chloride, and glyoxal were irradiated (Figure S1) and the decay of glyoxal was quantified using Raman peak of $v(\mathrm{C}-\mathrm{H})$ mode at $\sim 2950 \mathrm{~cm}^{-1}$. Note that the glyoxal oxidation loses the $\mathrm{C}-\mathrm{H}$ bounds ${ }^{8}$ and hence the $v(\mathrm{C}$ $\mathrm{H})$ peak is a good proxy for monitoring the oxidation. Figure S13 shows the decay in glyoxal represented by the peak at $v(\mathrm{C}-\mathrm{H})$ mode and the peak area at $1468 \mathrm{~cm}^{-1}$ as a function of time in the presence and absence of chlorine radicals. The presence of chlorine radicals shows faster decay in glyoxal than the absence, and the emergence of the peak at $1468 \mathrm{~cm}^{-1}$, whereas the absence does not exhibit the peak. The slop in the decay plot (Figure S13a) yields the apparent first-order reaction rate constants for the decay of glyoxal. The difference of the rate constants in the presence and absence of chlorine radicals is calculated to be $2.39 \times 10^{-6} \mathrm{~s}$, which is responsible for glyoxal oxidation by chlorine radicals. If the $\mathrm{Cl}$ concentration of $10^{-14} \mathrm{M}$ found in this study is taken, the intrinsic reaction rate constant of $\mathrm{Cl}$ and glyoxal is estimated to be $2.4 \times 10^{8} \mathrm{M}^{-1} \mathrm{~s}^{-1}$, comparable to the reported rate constants. ${ }^{9}$ 
Table S1. The observed concentrations of chloride, iron, sulfate, and carboxylic acids in fine particles across China

\begin{tabular}{|c|c|c|c|c|}
\hline \multirow{2}{*}{ Location } & \multicolumn{2}{|c|}{ Chloride } & \multirow{2}{*}{$\mathrm{Cl}^{-} / \mathrm{Na}^{+}$(mass ratio) } & \multirow{2}{*}{ Note } \\
\hline & $\mu \mathrm{g} \mathrm{m}^{-3}$ of air & $\mathrm{M}^{\mathrm{a}}$ & & \\
\hline Hainan & 1.8 & 0.85 & 3.0 & 10 \\
\hline Tongji & 1.7 & 0.80 & 2.4 & 10 \\
\hline Chegongzhuang & 1.6 & 0.75 & 2.3 & 10 \\
\hline Tsinghua & 1.8 & 0.85 & 2.6 & 10 \\
\hline Beijing & 3.06 & 1.44 & 5.00 & 11 \\
\hline Jinan & 3.12 & 1.47 & 3.10 & 11 \\
\hline Changchun & 2.55 & 1.20 & 3.11 & 11 \\
\hline Dalian & 1.96 & 0.92 & 1.32 & 11 \\
\hline Qingdao & 2.31 & 1.09 & 2.03 & 11 \\
\hline Nanjing & 1.61 & 0.76 & 1.45 & 11 \\
\hline Shanghai & 2.78 & 1.31 & 3.40 & 11 \\
\hline Hangzhou & 2.15 & 1.01 & 2.54 & 11 \\
\hline Xiamen & 1.40 & 0.66 & 1.75 & 11 \\
\hline Hong Kong & 0.77 & 0.36 & 1.06 & 11 \\
\hline Shenzhen & 1.55 & 0.73 & 0.38 & 11 \\
\hline Guangzhou & 1.02 & 0.48 & 0.85 & 11 \\
\hline Wuhan & 2.30 & 1.08 & 2.46 & 11 \\
\hline Chongqing & 4.93 & 2.32 & 4.17 & 11 \\
\hline Xi'an & 5.23 & 2.46 & 2.49 & 11 \\
\hline Jinchang & 2.65 & 1.25 & 1.67 & 11 \\
\hline Min & 0.77 & 0.36 & 0.38 & \\
\hline Max & 5.23 & 2.46 & 5 & \\
\hline Average & 2.31 & 1.09 & 2.35 & \\
\hline Location & $\begin{array}{l}\text { Total iron } \\
\left(\mathrm{ng} \mathrm{m}^{-3}\right)\end{array}$ & $\begin{array}{c}\text { Water } \\
\text { soluble iron } \\
\left(\mathrm{ng} \mathrm{m}^{-3}\right)^{\mathrm{b}}\end{array}$ & {$[\text { Iron }]_{\mathrm{aq}}(\mathrm{mM})^{\mathrm{a}}$} & Note \\
\hline Mt Lushan & 331.1 & 71.5 & 21.3 & 12 \\
\hline Beijing & & 18 & 5.37 & 13 \\
\hline Fuzhou & $655^{\mathrm{c}}$ & 33 & 9.85 & 14 \\
\hline Shanghai & 900 & 45 & 13.4 & 15 \\
\hline Yong'an & 749 & 37 & 11.0 & 16 \\
\hline Chengdu & 2871 & 144 & 43.0 & 17 \\
\hline Jinan & 1640 & 82 & 24.5 & 18 \\
\hline Beijing & 1150 & 58 & 17.3 & 19 \\
\hline Shanghai & 820 & 41 & 12.2 & 19 \\
\hline Zhengzhou & 1192 & 60 & 17.9 & 20 \\
\hline \multirow{3}{*}{\multicolumn{3}{|c|}{$\begin{array}{c}\text { Min } \\
\text { Max } \\
\text { Average }\end{array}$}} & 5.37 & \\
\hline & & & 43.0 & \\
\hline & & & 17.6 & \\
\hline
\end{tabular}


Table S1. Continued

\begin{tabular}{|c|c|c|c|c|c|c|c|}
\hline \multirow{2}{*}{ Location } & \multicolumn{6}{|c|}{ Sulfate } & \multirow{2}{*}{ Note } \\
\hline & \multicolumn{3}{|c|}{$\mu \mathrm{g} \mathrm{m}^{-3}$ of air } & \multicolumn{3}{|c|}{$\mathrm{M}^{\mathrm{a}}$} & \\
\hline Hainan & \multicolumn{3}{|c|}{15.9} & \multicolumn{3}{|c|}{2.76} & 10 \\
\hline Tongji & \multicolumn{3}{|c|}{15.2} & \multicolumn{3}{|c|}{2.64} & 10 \\
\hline Chegongzhuang & \multicolumn{3}{|c|}{18.4} & \multicolumn{3}{|c|}{3.19} & 10 \\
\hline Tsinghua & \multicolumn{3}{|c|}{16.9} & \multicolumn{3}{|c|}{2.93} & 10 \\
\hline Min & \multicolumn{3}{|c|}{15.2} & \multicolumn{3}{|c|}{2.64} & \\
\hline Max & \multicolumn{3}{|c|}{18.4} & \multicolumn{3}{|c|}{3.19} & \\
\hline Average & \multirow{2}{*}{\multicolumn{2}{|c|}{$\frac{17.2}{\text { Oxalate }}$}} & & \multicolumn{3}{|c|}{2.99} & \\
\hline \multirow[b]{2}{*}{ Location } & & & \multicolumn{2}{|c|}{ Malonate } & \multicolumn{2}{|c|}{ Succinate } & Note \\
\hline & $\begin{array}{l}\mu \mathrm{g} \mathrm{m}^{-3} \\
\text { of air }\end{array}$ & $\mathrm{mM}^{\mathrm{a}}$ & $\begin{array}{l}\mu g^{-3} \\
\text { of air }\end{array}$ & $\mathrm{mM}$ & $\begin{array}{l}\mu \mathrm{g} \mathrm{m}^{-3} \\
\text { of air }\end{array}$ & $\mathrm{mM}$ & \\
\hline Hainan & 0.5 & 94.7 & 0.04 & 6.53 & 0.02 & 2.82 & 10 \\
\hline Tongji & 0.5 & 94.7 & 0.1 & 16.3 & 0.02 & 2.82 & 10 \\
\hline Chegongzhuang & 0.3 & 56.8 & 0.1 & 16.3 & 0.03 & 4.23 & 10 \\
\hline Tsinghua & 0.3 & 56.8 & 0.1 & 16.3 & 0.02 & 2.82 & 10 \\
\hline $\begin{array}{l}\text { Tsim sha tsui } \\
\text { (Hong Kong) }\end{array}$ & 0.35 & 66.3 & 0.06 & 9.8 & 0.09 & 12.7 & 21 \\
\hline Min & 0.3 & 56.8 & 0.04 & 6.53 & 0.02 & 2.82 & \\
\hline Max & 0.5 & 94.7 & 0.1 & 16.3 & 0.09 & 12.7 & \\
\hline Average & 0.39 & 73.8 & 0.08 & 13.1 & 0.04 & 5.08 & \\
\hline
\end{tabular}

a) Aerosol liquid water content of $6 \times 10^{-8} \mathrm{~L} \mathrm{~m}^{-3}$ of air $^{22}$ was used to estimate the corresponding molar concentrations.

b) The lower limit of typical Fe solubility of $5 \%{ }^{23-25}$ was used to estimate the concentrations of water soluble iron.

c) Average value

Table S2. Reactions considered in this study

\begin{tabular}{|c|c|c|c|}
\hline & Reactions & Rate constant & Note \\
\hline \multicolumn{4}{|c|}{ Iron/chlorine/sulfur chemistry ${ }^{a}$} \\
\hline R1 & $\mathrm{FeCl}^{2+}+h v \rightarrow \mathrm{Fe}^{2+}+\mathrm{Cl}^{\bullet}$ & $j_{\mathrm{FeCl} 2+}$ & 26,27 \\
\hline $\mathrm{R} 2$ & $\mathrm{Fe}(\mathrm{Cl})_{2}{ }^{+}+h v \rightarrow \mathrm{FeCl}^{+}+\mathrm{Cl}^{-}$ & $\bar{j} j_{\mathrm{Fe}(\mathrm{Cl}) 2+}$ & 26,27 \\
\hline $\mathrm{R} 3$ & $\mathrm{FeSO}_{4}^{+}+h v \rightarrow \mathrm{Fe}^{2+}+\mathrm{SO}_{4}^{\cdot-}$ & $j_{\mathrm{R} 3}=0.0034 \times j_{\mathrm{FeCl} 2+}$ & 28 \\
\hline $\mathrm{R} 4^{\mathrm{a}}$ & $\mathrm{Fe}^{3+}+\mathrm{H}_{2} \mathrm{O} \rightleftharpoons \mathrm{FeOH}^{2+}+\mathrm{H}^{+}$ & $\begin{array}{l}k_{\mathrm{R} 4}=2.34 \times 10^{7} \mathrm{~s}^{-1} \\
k_{\mathrm{R} 4}=1 \times 10^{10} \mathrm{M}^{-1} \mathrm{~s}^{-1}\end{array}$ & 29 \\
\hline $\mathrm{R} 5$ & $\mathrm{Fe}^{3+}+2 \mathrm{H}_{2} \mathrm{O} \rightleftharpoons \mathrm{Fe}(\mathrm{OH})_{2}^{+}+\mathrm{H}^{+}$ & $\begin{array}{l}k_{\mathrm{R} 5}=4.68 \times 10^{3} \mathrm{~s}^{-1} \\
k_{\mathrm{R} 5}=1 \times 10^{10} \mathrm{M}^{-1} \mathrm{~s}^{-1}\end{array}$ & 29 \\
\hline R6 & $2 \mathrm{Fe}^{3+}+2 \mathrm{H}_{2} \mathrm{O} \rightleftharpoons \mathrm{Fe}_{2}(\mathrm{OH})_{2}{ }^{4+}+2 \mathrm{H}^{+}$ & $\begin{array}{l}k_{\mathrm{R} 6}=1.12 \times 10^{7} \mathrm{M}^{-1} \mathrm{~s}^{-1} \\
k_{-\mathrm{R} 6}=1 \times 10^{10} \mathrm{M}^{-2} \mathrm{~s}^{-1}\end{array}$ & 29 \\
\hline
\end{tabular}




\begin{tabular}{|c|c|c|c|}
\hline R7 & $\mathrm{Fe}^{3+}+\mathrm{Cl}^{-} \rightleftharpoons \mathrm{FeCl}^{2+}$ & $\begin{array}{l}k_{\mathrm{R} 7}=6.61 \times 10^{10} \mathrm{M}^{-1} \mathrm{~s}^{-1} \\
k_{\mathrm{R} 7}=1 \times 10^{10} \mathrm{~s}^{-1}\end{array}$ & 29 \\
\hline R8 & $\mathrm{Fe}^{3+}+2 \mathrm{Cl}^{-} \rightleftharpoons \mathrm{FeCl}_{2}^{+}$ & $\begin{array}{l}k_{\mathrm{R} 8}=1.05 \times 10^{11} \mathrm{M}^{-1} \mathrm{~s}^{-1} \\
k_{\mathrm{R} 8}=1 \times 10^{10} \mathrm{~s}^{-1}\end{array}$ & 29 \\
\hline R9 & $\mathrm{Fe}^{2+}+\mathrm{Cl}^{-} \rightleftharpoons \mathrm{FeCl}^{+}$ & $\begin{array}{l}k_{\mathrm{R} 9}=2.88 \times 10^{10} \mathrm{M}^{-1} \mathrm{~s}^{-1} \\
k_{-} \mathrm{R} 9=1 \times 10^{10} \mathrm{~s}^{-1}\end{array}$ & 29 \\
\hline $\mathrm{R} 10$ & $\mathrm{Cl}^{-}+\mathrm{HSO}_{3}^{-} \rightarrow \mathrm{Cl}^{-}+\mathrm{H}^{+}+\mathrm{SO}_{3}{ }^{--}$ & $k_{\mathrm{R} 10}=3.4 \times 10^{9} \mathrm{M}^{-1} \mathrm{~s}^{-1}$ & 30 \\
\hline R11 & $\mathrm{Cl}_{2}{ }^{--}+\mathrm{HSO}_{3}^{-} \rightarrow 2 \mathrm{Cl}^{-}+\mathrm{H}^{+}+\mathrm{SO}_{3}^{\cdot-}$ & $k_{\mathrm{R} 11}=3.4 \times 10^{8} \mathrm{M}^{-1} \mathrm{~s}^{-1}$ & 30 \\
\hline $\mathrm{R} 12$ & $\mathrm{SO}_{4}^{\bullet-}+\mathrm{Cl}^{-} \rightleftharpoons \mathrm{SO}_{4}^{2-}+\mathrm{Cl}^{\bullet}$ & $\begin{array}{l}k_{\mathrm{R} 12}=2.5 \times 10^{8} \mathrm{M}^{-1} \mathrm{~s}^{-1} \\
k_{-\mathrm{R} 12}=6.1 \times 10^{8} \mathrm{M}^{-1} \mathrm{~s}^{-1}\end{array}$ & 31 \\
\hline R13 & $\mathrm{SO}_{3}{ }^{\bullet-}+\mathrm{O}_{2} \rightarrow \mathrm{SO}_{5}{ }^{--}$ & $k_{\mathrm{R} 13}=1.5 \times 10^{9} \mathrm{M}^{-1} \mathrm{~s}^{-1}$ & 32 \\
\hline R14 & $\mathrm{SO}_{5}{ }^{--}+\mathrm{HSO}_{3}^{-} \rightarrow \mathrm{HSO}_{5}^{-}+\mathrm{SO}_{3}{ }^{--}$ & $k_{\mathrm{R} 14}=2.5 \times 10^{4} \mathrm{M}^{-1} \mathrm{~s}^{-1}$ & 32 \\
\hline $\mathrm{R} 15$ & $\mathrm{SO}_{5}{ }^{--}+\mathrm{HSO}_{3}^{-} \rightarrow \mathrm{SO}_{4}{ }^{--}+\mathrm{SO}_{4}^{2-}+\mathrm{H}^{+}$ & $k_{\mathrm{R} 15}=7.5 \times 10^{4} \mathrm{M}^{-1} \mathrm{~s}^{-1}$ & 32 \\
\hline R16 & $\mathrm{SO}_{4}^{--}+\mathrm{HSO}_{3}^{-} \rightarrow \mathrm{SO}_{4}^{2-}+\mathrm{SO}_{3}{ }^{--}+\mathrm{H}^{+}$ & $k_{\mathrm{R} 16}=7.5 \times 10^{8} \mathrm{M}^{-1} \mathrm{~s}^{-1}$ & 32 \\
\hline $\mathrm{R} 17$ & $2 \mathrm{SO}_{5}{ }^{--} \rightarrow 2 \mathrm{SO}_{4}^{-}+\mathrm{O}_{2}$ & $k_{\mathrm{R} 17}=6 \times 10^{8} \mathrm{M}^{-1} \mathrm{~s}^{-1}$ & 32 \\
\hline $\mathrm{R} 18$ & $2 \mathrm{SO}_{3}{ }^{--} \rightarrow \mathrm{S}_{2} \mathrm{O}_{6}{ }^{2-}$ & $k_{\mathrm{R} 18}=7 \times 10^{8} \mathrm{M}^{-1} \mathrm{~s}^{-1}$ & 32 \\
\hline R19 & $2 \mathrm{SO}_{4}{ }^{--} \rightarrow \mathrm{S}_{2} \mathrm{O}_{8}{ }^{2-}$ & $k_{\mathrm{R} 19}=4.5 \times 10^{8} \mathrm{M}^{-1} \mathrm{~s}^{-1}$ & 32 \\
\hline $\mathrm{R} 20$ & $2 \mathrm{SO}_{5}{ }^{--} \rightarrow \mathrm{S}_{2} \mathrm{O}_{8}^{2-}+\mathrm{O}_{2}$ & $k_{\mathrm{R} 20}=1.4 \times 10^{8} \mathrm{M}^{-1} \mathrm{~s}^{-1}$ & 32 \\
\hline $\mathrm{R} 21$ & $\mathrm{HSO}_{5}^{-}+\mathrm{HSO}_{3}^{-}+\mathrm{H}^{+} \rightarrow 2 \mathrm{SO}_{4}^{2-}+3 \mathrm{H}^{+}$ & $k_{\mathrm{R} 21}=7.1 \times 10^{6} \mathrm{M}^{-2} \mathrm{~s}^{-1}$ & 32 \\
\hline $\mathrm{R} 22$ & $2 \mathrm{SO}_{3}^{--} \rightarrow \mathrm{SO}_{3}+\mathrm{SO}_{3}^{2-}$ & $k_{\mathrm{R} 22}=3.17 \times 10^{8} \mathrm{M}^{-1} \mathrm{~s}^{-1}$ & 32 \\
\hline R23 & $\mathrm{Cl}^{\bullet}+\mathrm{H}_{2} \mathrm{O} \rightleftharpoons \mathrm{ClOH}^{-}+\mathrm{H}^{+}$ & $\begin{array}{l}k_{\mathrm{R} 23}=1.8 \times 10^{5} \mathrm{M}^{-1} \mathrm{~s}^{-1} \\
k_{\mathrm{R} 23}=2.4 \times 10^{10} \mathrm{M}^{-1} \mathrm{~s}^{-1}\end{array}$ & 29 \\
\hline $\mathrm{R} 24$ & $\mathrm{ClOH}^{-} \rightleftharpoons \mathrm{Cl}^{-}+\mathrm{OH}$ & $\begin{array}{l}k_{\mathrm{R} 24}=6.0 \times 10^{9} \mathrm{~s}^{-1} \\
k_{\mathrm{R} 24}=4.2 \times 10^{9} \mathrm{M}^{-1} \mathrm{~s}^{-1}\end{array}$ & 29 \\
\hline $\mathrm{R} 25$ & $\mathrm{Cl}^{\bullet}+\mathrm{Cl}^{-} \rightleftharpoons \mathrm{Cl}_{2}{ }^{\bullet}$ & $\begin{array}{l}k_{\mathrm{R} 25}=7.8 \times 10^{9} \mathrm{M}^{-1} \mathrm{~s}^{-1} \\
k_{\mathrm{R} 25}=5.7 \times 10^{4} \mathrm{~s}^{-1}\end{array}$ & 29 \\
\hline R26 & $\mathrm{Cl}_{2}{ }^{\bullet-}+\mathrm{H}_{2} \mathrm{O} \rightarrow \mathrm{ClOH}^{-}+\mathrm{Cl}^{-}+\mathrm{H}^{+}$ & $k_{\mathrm{R} 26}=1.3 \times 10^{3} \mathrm{~s}^{-1}$ & 29 \\
\hline R27 & $\mathrm{Fe}^{3+}+\mathrm{SO}_{4}^{2-} \rightleftharpoons \mathrm{FeSO}_{4}^{+}$ & $\begin{array}{l}k_{\mathrm{R} 27}=2.32 \times 10^{12} \mathrm{M}^{-1} \mathrm{~s}^{-1} \\
k \_\mathrm{R} 27=1 \times 10^{10} \mathrm{~s}^{-1}\end{array}$ & 31 \\
\hline R28 & $\mathrm{Fe}^{3+}+2 \mathrm{SO}_{4}^{2-} \rightleftharpoons \mathrm{Fe}\left(\mathrm{SO}_{4}\right)_{2}^{-}$ & $\begin{array}{l}k_{\mathrm{R} 28}=3.29 \times 10^{13} \mathrm{M}^{-1} \mathrm{~s}^{-1} \\
k_{\mathrm{R} 28}=1 \times 10^{10} \mathrm{~s}^{-1}\end{array}$ & 31 \\
\hline $\mathrm{R} 29$ & $\mathrm{Fe}^{2+}+\mathrm{SO}_{4}{ }^{2-} \rightleftharpoons \mathrm{FeSO}_{4}$ & $\begin{array}{l}k_{\mathrm{R} 29}=1.19 \times 10^{11} \mathrm{M}^{-1} \mathrm{~s}^{-1} \\
k_{\mathrm{R} 29}=1 \times 10^{10} \mathrm{~s}^{-1}\end{array}$ & 31 \\
\hline R30 & $\mathrm{H}^{+}+\mathrm{SO}_{4}^{2-} \rightleftharpoons \mathrm{HSO}_{4}^{-}$ & $\begin{array}{l}k_{\mathrm{R} 30}=1.45 \times 10^{11} \mathrm{M}^{-1} \mathrm{~s}^{-1} \\
k_{-\mathrm{R} 30}=1 \times 10^{10} \mathrm{~s}^{-1}\end{array}$ & 31 \\
\hline R31 & $\mathrm{Cl}^{-}+\mathrm{Fe}^{2+} \rightarrow \mathrm{Cl}^{-}+\mathrm{Fe}^{3+}$ & $k_{\mathrm{R} 31}=5.9 \times 10^{9} \mathrm{M}^{-1} \mathrm{~s}^{-1}$ & 29 \\
\hline R32 & $\mathrm{Cl}_{2}{ }^{--}+\mathrm{Fe}^{2+} \rightarrow 2 \mathrm{Cl}^{-}+\mathrm{Fe}^{3+}$ & $k_{\mathrm{R} 32}=5 \times 10^{6} \mathrm{M}^{-1} \mathrm{~s}^{-1}$ & 29 \\
\hline R33 & $\mathrm{Fe}^{2+}+\mathrm{SO}_{4}{ }^{--} \rightarrow \mathrm{Fe}^{3+}+\mathrm{SO}_{4}^{2-}$ & $k_{\mathrm{R} 33}=3.0 \times 10^{8} \mathrm{M}^{-1} \mathrm{~s}^{-1}$ & 31 \\
\hline R34 & $\mathrm{Fe}^{2+}+\mathrm{HSO}_{5}^{-} \rightarrow \mathrm{FeOH}^{2+}+\mathrm{SO}_{4}^{\cdot-}$ & $k_{\mathrm{R} 34}=3 \times 10^{4} \mathrm{M}^{-1} \mathrm{~s}^{-1}$ & 33 \\
\hline R35 & $\mathrm{Fe}^{2+}+\mathrm{SO}_{5}{ }^{--}+\mathrm{H}_{2} \mathrm{O} \rightarrow \mathrm{FeOH}^{2+}+\mathrm{HSO}_{5}^{-}$ & $k_{\mathrm{R} 35}=1.16 \times 10^{4 \mathrm{~d}} \mathrm{M}^{-1} \mathrm{~s}^{-1}$ & 34 \\
\hline R36 & $\mathrm{Fe}^{2+} \stackrel{\mathrm{S}(\mathrm{IV}), \mathrm{O}_{2}}{\longrightarrow} \mathrm{Fe}^{3+}+\mathrm{O}_{2}^{\cdot-}$ & $k_{\mathrm{R} 36}=(5.7-6.1) \times 10^{-2} \mathrm{~s}^{-1 \mathrm{~d}}$ & 33 \\
\hline R37 & $\mathrm{HO}_{2}^{\bullet} \rightleftharpoons \mathrm{O}_{2}^{-\bullet}+\mathrm{H}^{+}$ & $\begin{array}{l}k_{\mathrm{R} 37}=1.58 \times 10^{5} \mathrm{~s}^{-1} \\
k_{\_} \mathrm{R} 37 \\
=1.0 \times 10^{10} \mathrm{M}^{-1} \mathrm{~s}^{-1}\end{array}$ & 29 \\
\hline R38 & $2 \mathrm{HO}_{2} \rightarrow \mathrm{H}_{2} \mathrm{O}_{2}+\mathrm{O}_{2}$ & $k_{\mathrm{R} 38}=8.3 \times 10^{5} \mathrm{M}^{-1} \mathrm{~s}^{-1}$ & 32 \\
\hline R39 & $\mathrm{S}(\mathrm{IV})+\mathrm{H}_{2} \mathrm{O}_{2} \rightarrow \mathrm{SO}_{4}^{2-}+\mathrm{H}_{2} \mathrm{O}$ & $\begin{array}{l}k_{\mathrm{R} 39}=7.45 \times 10^{7} \times \\
{[\mathrm{S}(\mathrm{IV})]\left[\mathrm{H}_{2} \mathrm{O}_{2}\right]\left[\mathrm{H}^{+}\right] /(1+13[} \\
\left.\left.\mathrm{H}^{+}\right]\right) \mathrm{M}^{-1} \mathrm{~s}^{-1}\end{array}$ & 32 \\
\hline
\end{tabular}


Nitrate chemistry

\begin{tabular}{|c|c|c|c|}
\hline $\mathrm{R} 40$ & $\begin{array}{l}2 \mathrm{Fe}^{2+}+\mathrm{NO}_{3}^{-}+2 \mathrm{H}^{+} \rightarrow 2 \mathrm{Fe}^{3+}+\mathrm{NO}_{2}^{-}+ \\
\mathrm{H}_{2} \mathrm{O}\end{array}$ & $k_{\mathrm{R} 40}=2.5 \times 10^{-2} \mathrm{M}^{-2} \mathrm{~s}^{-1}$ & 3,4 \\
\hline R41 & $\mathrm{NO}_{3}^{-}+h v \rightarrow{ }^{-} \mathrm{NO}_{2}+{ }^{\bullet} \mathrm{OH}$ & $\begin{array}{l}j_{\mathrm{R} 41}=1.1 \times 10^{-10}-9.0 \times \\
10^{-7} \mathrm{~s}^{-1 \mathrm{~d}}\end{array}$ & 35 \\
\hline $\mathrm{R} 42$ & $\mathrm{NO}_{3}^{-}+h v \rightarrow \mathrm{NO}_{2}^{-}+\mathrm{O}\left({ }^{3} \mathrm{P}\right)$ & $j_{\mathrm{R} 42}=(3.8-9.0) \times 10^{-7} \mathrm{~s}^{-1 \mathrm{~d}}$ & 35 \\
\hline $\mathrm{R} 43$ & $\mathrm{NO}_{2}^{-}+h v \rightarrow \mathrm{NO}+{ }^{-} \mathrm{OH}$ & $j_{\mathrm{R} 43}=(6.8-9.2) \times 10^{-3} \mathrm{~s}^{-1 \mathrm{~d}}$ & 35 \\
\hline $\mathrm{R} 44$ & $\begin{array}{l}\mathrm{NO}_{2}^{-}+\mathrm{S}(\mathrm{IV})+\mathrm{H}^{+} \rightarrow \mathrm{NOH}+\mathrm{SO}_{4}^{2-}+ \\
2 \mathrm{H}^{+}\end{array}$ & $k_{\mathrm{R} 44}=3.8 \times 10^{3} \mathrm{M}^{-2} \mathrm{~s}^{-1}$ & 36,37 \\
\hline $\mathrm{R} 45$ & $\begin{array}{l}2 \mathrm{NO}_{2}+\mathrm{S}(\mathrm{IV})+\mathrm{H}_{2} \mathrm{O} \rightarrow 3 \mathrm{H}^{+}+2 \mathrm{NO}_{2}^{-}+ \\
\mathrm{SO}_{4}^{2-}\end{array}$ & $k_{\mathrm{R} 45}=2.0 \times 10^{6} \mathrm{M}^{-1} \mathrm{~s}^{-1}$ & 32 \\
\hline R46 & $\mathrm{NO}_{3}^{-}+\mathrm{Cl}^{\bullet} \rightleftharpoons \mathrm{NO}_{3}+\mathrm{Cl}^{-}$ & $\begin{array}{l}k_{\mathrm{R} 46}=1.0 \times 10^{8} \mathrm{M}^{-1} \mathrm{~s}^{-1} \\
k_{\mathrm{R} 46}=3.5 \times 10^{8} \mathrm{M}^{-1} \mathrm{~s}^{-1}\end{array}$ & 32 \\
\hline \multicolumn{4}{|c|}{ Oxalate chemistry ${ }^{\mathrm{b}}$} \\
\hline $\mathrm{R} 47$ & $\mathrm{OA} \rightleftharpoons \mathrm{Ox}^{-}+\mathrm{H}^{+}$ & $\begin{array}{l}k_{\mathrm{R} 47}=5.32 \times 10^{8} \mathrm{~s}^{-1} \\
k_{-\mathrm{R} 47}=1 \times 10^{10} \mathrm{M}^{-1} \mathrm{~s}^{-1}\end{array}$ & 38 \\
\hline $\mathrm{R} 48$ & $\mathrm{Ox}^{-} \rightleftharpoons \mathrm{Ox}^{2-}+\mathrm{H}^{+}$ & $\begin{array}{l}k_{\mathrm{R} 48}=1.53 \times 10^{6} \mathrm{~s}^{-1} \\
k \_\mathrm{R} 48=1 \times 10^{10} \mathrm{M}^{-1} \mathrm{~s}^{-1}\end{array}$ & 38 \\
\hline R49 & $\mathrm{Fe}^{3+}+\mathrm{Ox}^{2-} \rightleftharpoons \mathrm{FeOx}^{+}$ & $\begin{array}{l}k_{\mathrm{R} 49}=5.89 \times 10^{18} \mathrm{M}^{-1} \mathrm{~s}^{-1} \\
k \_\mathrm{R} 49=1 \times 10^{10} \mathrm{~s}^{-1}\end{array}$ & 39 \\
\hline R50 & $\mathrm{FeOx}^{+}+\mathrm{Ox}_{2}^{-} \rightleftharpoons \mathrm{FeOx}_{2}^{-}$ & $\begin{array}{l}k_{\mathrm{R} 50}=3.31 \times 10^{16} \mathrm{M}^{-1} \mathrm{~s}^{-1} \\
k_{\mathrm{R} 50}=1 \times 10^{10} \mathrm{~s}^{-1}\end{array}$ & 39 \\
\hline R51 & $\mathrm{FeOx}_{2}^{-}+\mathrm{Ox}_{2}^{-} \rightleftharpoons \mathrm{FeOx}_{3}^{3-}$ & $\begin{array}{l}k_{\mathrm{R} 51}=2.75 \times 10^{14} \mathrm{M}^{-1} \mathrm{~s}^{-1} \\
k_{-\mathrm{R} 51}=1 \times 10^{10} \mathrm{~s}^{-1}\end{array}$ & 39 \\
\hline R52 & $\mathrm{FeOx}_{3}^{3-}+h v \rightarrow \mathrm{Fe}\left(\mathrm{C}_{2} \mathrm{O}_{4}\right)_{2}{ }^{2-}+\mathrm{C}_{2} \mathrm{O}_{4}^{-}$ & $j_{\mathrm{R} 52}=(1.7-2.9) \times 10^{-2} \mathrm{~s}^{-1 \mathrm{~d}}$ & 39,40 \\
\hline R53 & $\mathrm{C}_{2} \mathrm{O}_{4}^{-} \rightarrow \mathrm{CO}_{2}^{--}+\mathrm{CO}_{2}$ & $k_{\mathrm{R} 53}=2 \times 10^{6} \mathrm{~s}^{-1}$ & 40 \\
\hline $\mathrm{R} 54$ & $\mathrm{CO}_{2}{ }^{\bullet-}+\mathrm{O}_{2} \rightarrow \mathrm{O}_{2}^{\bullet-}+\mathrm{CO}_{2}$ & $k_{\mathrm{R} 54}=6.5 \times 10^{9} \mathrm{M}^{-1} \mathrm{~s}^{-1}$ & 40 \\
\hline R55 & $\mathrm{OA}+\mathrm{Cl}^{\cdot} \rightarrow$ product $+\mathrm{Cl}^{-}$ & $k_{\mathrm{R} 55}=1 \times 10^{9} \mathrm{M}^{-1} \mathrm{~s}^{-1 \mathrm{e}}$ & 9,41 \\
\hline R56 & $\mathrm{OA}+\mathrm{Cl}_{2}{ }^{-} \rightarrow$ product $+2 \mathrm{Cl}^{-}$ & $k_{\mathrm{R} 56}=1 \times 10^{6 \mathrm{e}} \mathrm{M}^{-1} \mathrm{~s}^{-1 \mathrm{e}}$ & 9,41 \\
\hline \multicolumn{4}{|c|}{ Malonate chemistry ${ }^{\mathrm{c}}$} \\
\hline R57 & $\mathrm{MA} \rightleftharpoons \mathrm{Mal}^{-}+\mathrm{H}^{+}$ & $\begin{array}{l}k_{\mathrm{R} 57}=2.06 \times 10^{7} \mathrm{~s}^{-1} \\
k_{-\mathrm{R} 57}=1 \times 10^{10} \mathrm{M}^{-1} \mathrm{~s}^{-1}\end{array}$ & 42 \\
\hline $\mathrm{R} 58$ & $\mathrm{Mal}^{-} \rightleftharpoons \mathrm{Mal}^{2-}+\mathrm{H}^{+}$ & $\begin{array}{l}k_{\mathrm{R} 58}=2.94 \times 10^{4} \mathrm{~s}^{-1} \\
k_{\mathrm{R} 58}=1 \times 10^{10} \mathrm{M}^{-1} \mathrm{~s}^{-1}\end{array}$ & 42 \\
\hline R59 & $\mathrm{Fe}^{3+}+\mathrm{Mal}^{2-} \rightleftharpoons \mathrm{FeMal}^{+}$ & $\begin{array}{l}k_{\mathrm{R} 59}=3.16 \times 10^{17} \mathrm{M}^{-1} \mathrm{~s}^{-1} \\
k_{-\mathrm{R} 59}=1 \times 10^{10} \mathrm{~s}^{-1}\end{array}$ & 43 \\
\hline R60 & $\mathrm{FeMal}^{+}+\mathrm{Mal}^{2-} \rightleftharpoons \mathrm{FeMal}_{2}^{-}$ & $\begin{array}{l}k_{\mathrm{R} 60}=6.31 \times 10^{15} \mathrm{M}^{-1} \mathrm{~s}^{-1} \\
k_{\mathrm{R} 60}=1 \times 10^{10} \mathrm{~s}^{-1}\end{array}$ & 43 \\
\hline R61 & $\mathrm{FeMal}_{2}^{-}+\mathrm{Mal}^{2-} \rightleftharpoons \mathrm{FeMal}_{3}^{3-}$ & $\begin{array}{l}k_{\mathrm{R} 61}=3.98 \times 10^{13} \mathrm{M}^{-1} \mathrm{~s}^{-1} \\
k_{-\mathrm{R} 61}=1 \times 10^{10} \mathrm{~s}^{-1}\end{array}$ & 43 \\
\hline R62 & $\mathrm{FeMal}^{+}+h v \rightarrow \mathrm{Fe}(\mathrm{II})-\left(\mathrm{CH}_{2} \mathrm{COO}\right)+\mathrm{CO}_{2}$ & $\underset{\mathrm{d}}{j_{\mathrm{R} 62}}=(0.42-2.5) \times 10^{-4} \mathrm{~s}^{-1}$ & 5 \\
\hline R63 & $\begin{array}{l}\mathrm{FeMal}_{2}^{-}+\mathrm{MA} / \mathrm{Mal}^{-}+h v \rightarrow \mathrm{Fe}(\mathrm{II})- \\
\left(\mathrm{Mal}^{-}\right)\left(\mathrm{CH}_{2} \mathrm{COO}\right)+\mathrm{CO}_{2}\end{array}$ & $j_{\mathrm{d} 62}=(0.42-2.5) \times 10^{-4} \mathrm{~s}^{-1}$ & 5 \\
\hline R64 & 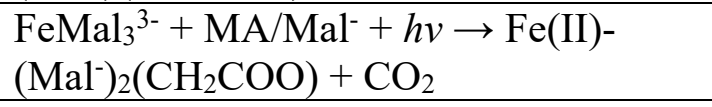 & $\underset{\mathrm{d} 62}{j_{\mathrm{d}}}=(0.42-2.5) \times 10^{-4} \mathrm{~s}^{-1}$ & 5 \\
\hline
\end{tabular}




\begin{tabular}{|c|c|c|c|}
\hline R65 & $\begin{array}{l}\mathrm{Fe}(\mathrm{II})-\left(\mathrm{CH}_{2} \mathrm{COO}\right)+\mathrm{MA} / \mathrm{Mal}^{-} \rightarrow \mathrm{FeMal}^{+} \\
+\mathrm{CH}_{3} \mathrm{COOH}\end{array}$ & $k_{\mathrm{R} 65}=1 \times 10^{9} \mathrm{M}^{-1} \mathrm{~s}^{-1 \mathrm{f}}$ & 5 \\
\hline R66 & $\begin{array}{l}\mathrm{Fe}(\mathrm{II})-\left(\mathrm{Mal}^{-}\right)\left(\mathrm{CH}_{2} \mathrm{COO}\right)+\mathrm{MA} / \mathrm{Mal}^{-} \rightarrow \\
\mathrm{FeMal}_{2}^{-}+\mathrm{CH}_{3} \mathrm{COOH}\end{array}$ & $k_{\mathrm{R} 66}=1 \times 10^{9} \mathrm{M}^{-1} \mathrm{~s}^{-1 \mathrm{f}}$ & 5 \\
\hline R67 & $\begin{array}{l}\mathrm{Fe}(\mathrm{II})-\left(\mathrm{Mal}^{-}\right)_{2}\left(\mathrm{CH}_{2} \mathrm{COO}\right)+\mathrm{MA} / \mathrm{Mal}^{-} \rightarrow \\
\mathrm{FeMal}_{3}^{3-}+\mathrm{CH}_{3} \mathrm{COOH}\end{array}$ & $k_{\mathrm{R} 67}=1 \times 10^{9} \mathrm{M}^{-1} \mathrm{~s}^{-1 \mathrm{f}}$ & 5 \\
\hline R68 & $\mathrm{MA}+\mathrm{Cl}^{\bullet} \rightarrow$ product $+\mathrm{Cl}^{-}$ & $k_{\mathrm{R} 68}=1 \times 10^{9} \mathrm{M}^{-1} \mathrm{~s}^{-1 \mathrm{e}}$ & 9,41 \\
\hline R69 & $\mathrm{MA}+\mathrm{Cl}_{2}{ }^{--} \rightarrow$ product $+\mathrm{Cl}^{-}$ & $k_{\mathrm{R} 69}=1 \times 10^{6} \mathrm{M}^{-1} \mathrm{~s}^{-1 \mathrm{e}}$ & 9,41 \\
\hline
\end{tabular}

a) $\mathrm{Fe}^{3+}=\mathrm{Fe}\left(\mathrm{H}_{2} \mathrm{O}\right)_{6}{ }^{3+} ; \mathrm{Fe}^{2+}=\mathrm{Fe}\left(\mathrm{H}_{2} \mathrm{O}\right)_{6}{ }^{2+} ; \mathrm{Fe}(\mathrm{OH})^{2+}=\left(\mathrm{H}_{2} \mathrm{O}\right)_{5} \mathrm{FeOH}^{2+} ; \mathrm{Fe}(\mathrm{OH})_{2}{ }^{+}=$

$\left(\mathrm{H}_{2} \mathrm{O}\right)_{4} \mathrm{Fe}(\mathrm{OH})_{2}{ }^{+} ; \mathrm{Fe}_{2}(\mathrm{OH})_{2}{ }^{4+}=2\left[\left(\mathrm{H}_{2} \mathrm{O}\right)_{5} \mathrm{Fe}(\mathrm{OH})\right] ; \mathrm{FeCl}^{2+}=\left(\mathrm{H}_{2} \mathrm{O}\right)_{5} \mathrm{FeCl}^{2+} ; \mathrm{FeCl}_{2}{ }^{+}=$

$\left(\mathrm{H}_{2} \mathrm{O}\right)_{4} \mathrm{FeCl}_{2}^{+}$

b) Oxalic acid $\left(\mathrm{OA} ; \mathrm{C}_{2} \mathrm{H}_{2} \mathrm{O}_{4}\right)$, hydrogen oxalate $\left(\mathrm{Ox}^{-} ; \mathrm{C}_{2} \mathrm{HO}_{4}^{-}\right)$, oxalate $\left(\mathrm{Ox}^{2-} ; \mathrm{C}_{2} \mathrm{O}_{4}{ }^{2-}\right)$

c) Malonic acid $\left(\mathrm{MA} ; \mathrm{C}_{3} \mathrm{H}_{4} \mathrm{O}_{4}\right)$, hydrogen malonate $\left(\mathrm{Mal}^{-} ; \mathrm{C}_{3} \mathrm{H}_{3} \mathrm{O}_{4}^{-}\right)$, malonate $\left(\mathrm{Mal}^{2-}\right.$;

$\left.\mathrm{C}_{3} \mathrm{H}_{2} \mathrm{O}_{4}{ }^{2-}\right)$

d) Based on the best model fit

e) Assumed based on the literature

f) Assumed to be very quick

Table S3. Initial conditions of each experiment and results of model fitting to experimentally determined sulfate production

\begin{tabular}{|c|c|c|c|c|c|}
\hline Experiment & {$\left[\mathrm{Fe}^{3+}\right](\mathrm{mM})$} & {$\left[\mathrm{Cl}^{-}\right](\mathrm{M})$} & {$\left[\mathrm{NO}^{3-}\right](\mathrm{M})$} & \multicolumn{2}{|c|}{$j_{\mathrm{FeCl} 2+}$ or $j_{\mathrm{Fe}(\mathrm{Cl}) 2+}\left(\mathrm{s}^{-1}\right)$} \\
\hline 1 & 5.1 & 5.1 & 0 & \multicolumn{2}{|c|}{4.1} \\
\hline 2 & 5.7 & 2.9 & 2.9 & \multicolumn{2}{|c|}{$6.5 \times 10^{-1}$} \\
\hline 3 & 6.1 & 1.5 & 4.5 & \multicolumn{2}{|c|}{$1.8 \times 10^{-1}$} \\
\hline 4 & 2.9 & 2.9 & 2.9 & \multicolumn{2}{|c|}{$8.3 \times 10^{-1}$} \\
\hline 5 & 0.57 & 2.9 & 2.9 & \multicolumn{2}{|c|}{$1.1 \times 10^{-2}$} \\
\hline 6 & 0.057 & 2.9 & 2.9 & \multicolumn{2}{|c|}{$4.5 \times 10^{-3}$} \\
\hline Experiment & {$\left[\mathrm{Fe}^{3+}\right](\mathrm{mM})$} & {$\left[\mathrm{Cl}^{-}\right](\mathrm{M})$} & {$[\mathrm{OA}](\mathrm{M})$} & $j_{\mathrm{FeCl} 2+}$ or $j_{\mathrm{Fe}(\mathrm{Cl}) 2+}\left(\mathrm{s}^{-1}\right)$ & $j_{\mathrm{R} 52}\left(\mathrm{~s}^{-1}\right)$ \\
\hline 7 & 5.4 & 4.1 & 1.4 & 3.1 & $1.7 \times 10^{-2}$ \\
\hline 8 & 7.6 & 3.8 & 3.8 & 1.8 & $2.9 \times 10^{-2}$ \\
\hline Experiment & {$\left[\mathrm{Fe}^{3+}\right](\mathrm{mM})$} & {$\left[\mathrm{Cl}^{-}\right](\mathrm{M})$} & [MA] (M) & $j_{\mathrm{FeCl} 2}+$ or $j_{\mathrm{Fe}(\mathrm{Cl}) 2+}\left(\mathrm{s}^{-1}\right)$ & $j_{\mathrm{R} 62}, j_{\mathrm{R} 63}$, or $j_{\mathrm{R} 64}\left(\mathrm{~s}^{-1}\right)$ \\
\hline 9 & 6.1 & 3.0 & 3.0 & 23.4 & $4.2 \times 10^{-5}$ \\
\hline 10 & 5.5 & 4.1 & 1.4 & 23.4 & $2.5 \times 10^{-4}$ \\
\hline
\end{tabular}


Table S4. Molar absorptivity and quantum yield of photolytic species considered in this study

\begin{tabular}{|c|c|c|c|}
\hline $\begin{array}{c}\text { Photolytic } \\
\text { species }\end{array}$ & $\varepsilon\left(\mathrm{M}^{-1} \mathrm{~cm}^{-1}\right)$ & $\Phi\left(\mathrm{Fe}^{2+}\right)$ & Note/reference \\
\hline $\mathrm{NO}_{3}{ }^{-}$ & $\begin{array}{c}7.02 \text { at } 300 \mathrm{~nm} ; \\
0.018 \text { at } 350 \mathrm{~nm}\end{array}$ & $\begin{array}{c}0.01 \text { for } \mathrm{NO}_{2}, \mathrm{OH}, \text { and } \mathrm{NO}_{2}{ }^{-} \\
\text {at }>300 \mathrm{~nm}\end{array}$ & 44 \\
\hline $\mathrm{FeCl}^{2+}$ & 1600 at $356 \mathrm{~nm}$ & 0.47 at $347 \mathrm{~nm}$ & 27,45 \\
\hline $\mathrm{FeCl}_{2}{ }^{+}$ & 2740 at $356 \mathrm{~nm}$ & N.A. & 27,45 \\
\hline $\mathrm{FeSO}_{4}{ }^{+}$ & 576 at $350 \mathrm{~nm}$ & 0.0016 at $350 \mathrm{~nm}$ & 28 \\
\hline $\mathrm{Fe}(\mathrm{III})-$-oxalato & $1040-1120$ at $351 \mathrm{~nm}$ & 0.88 at $351 \mathrm{~nm}$ & 40 \\
\hline $\mathrm{Fe}(\mathrm{III})-$ malonato & 210 at $351 \mathrm{~nm}$ & 0.0022 at $300-366 \mathrm{~nm}$ & 5 \\
\hline
\end{tabular}




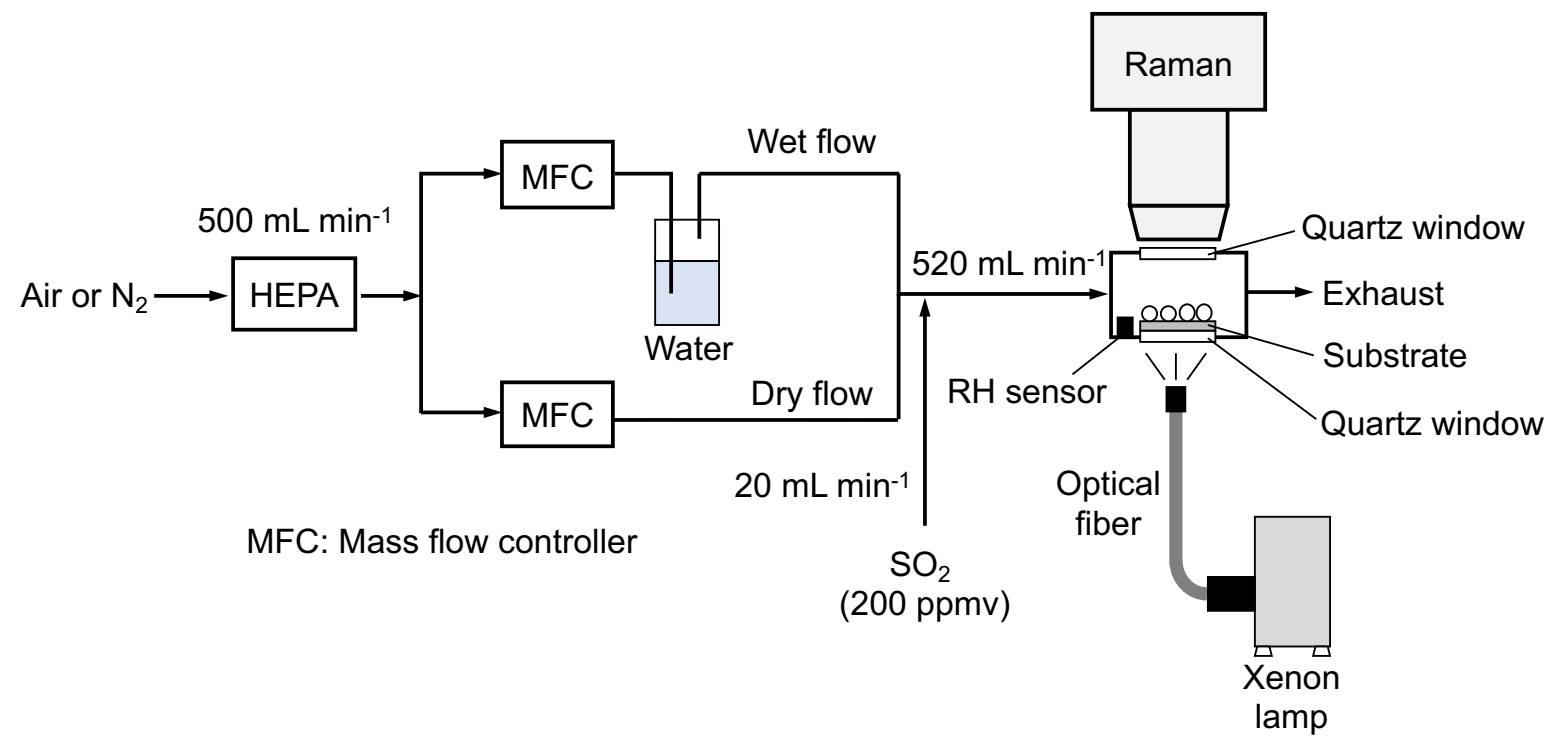

Figure S1. Schematic illustration of experimental setup used in this study.
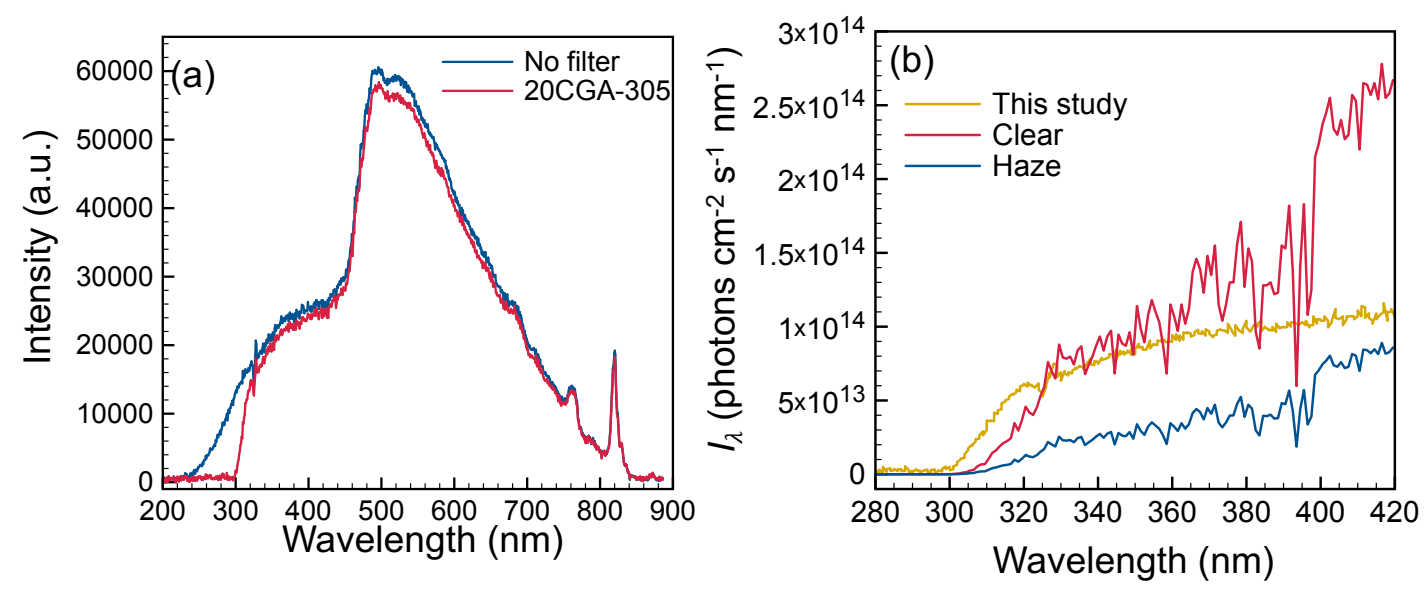

Figure S2. (a) Wavelength-dependent intensity of irradiation in the presence and absence of the optical filter (20CGA-305). (b) Photon fluxes of irradiation with the optical filter used in this study in comparison with typical photon fluxes in Beijing on clear and haze days. 

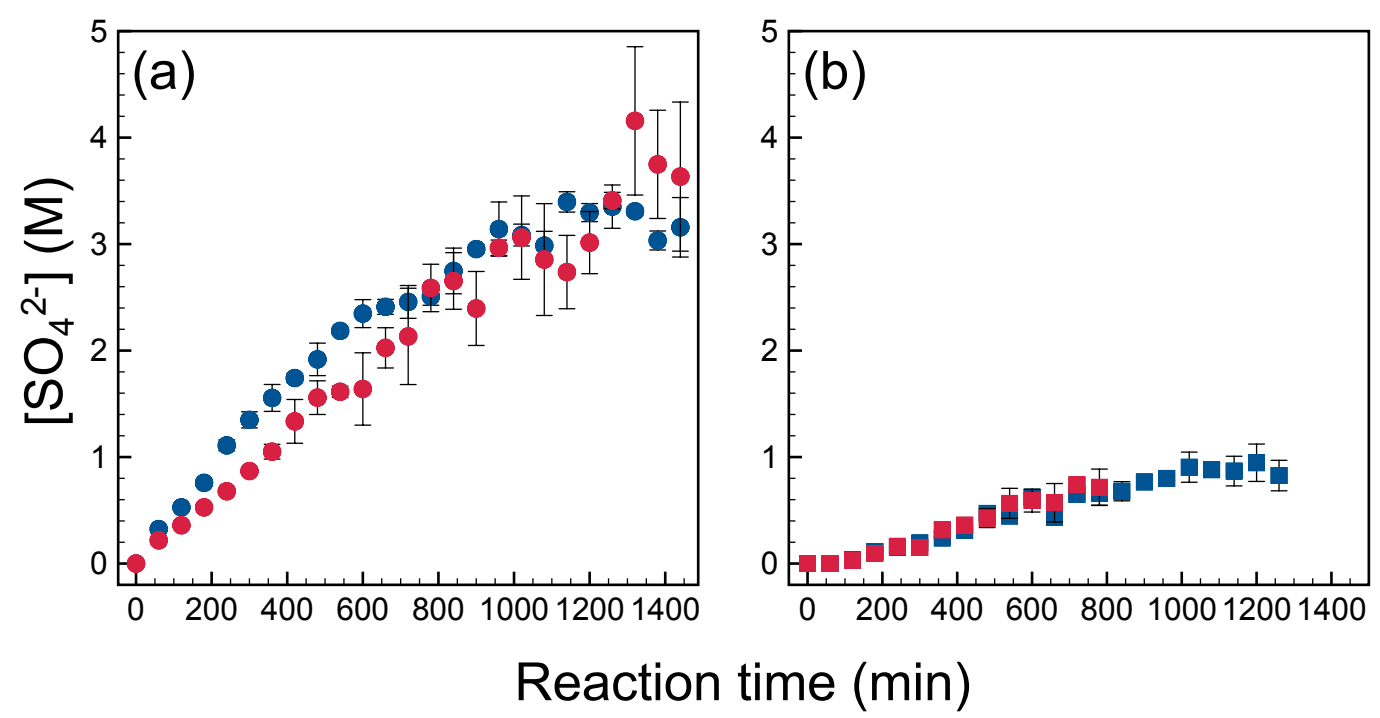

Figure S3. Replicate experiments for multiphase oxidation of $\mathrm{SO}_{2}$ by irradiated particles containing (a) $\mathrm{Cl}^{-}$and $\mathrm{Fe}^{3+}$, and (b) $\mathrm{NO}_{3}{ }^{-}$and $\mathrm{Fe}^{3+}$. The first and repeated experiments are in blue and red, respectively. The error bars represent one standard deviation from three Raman measurements.

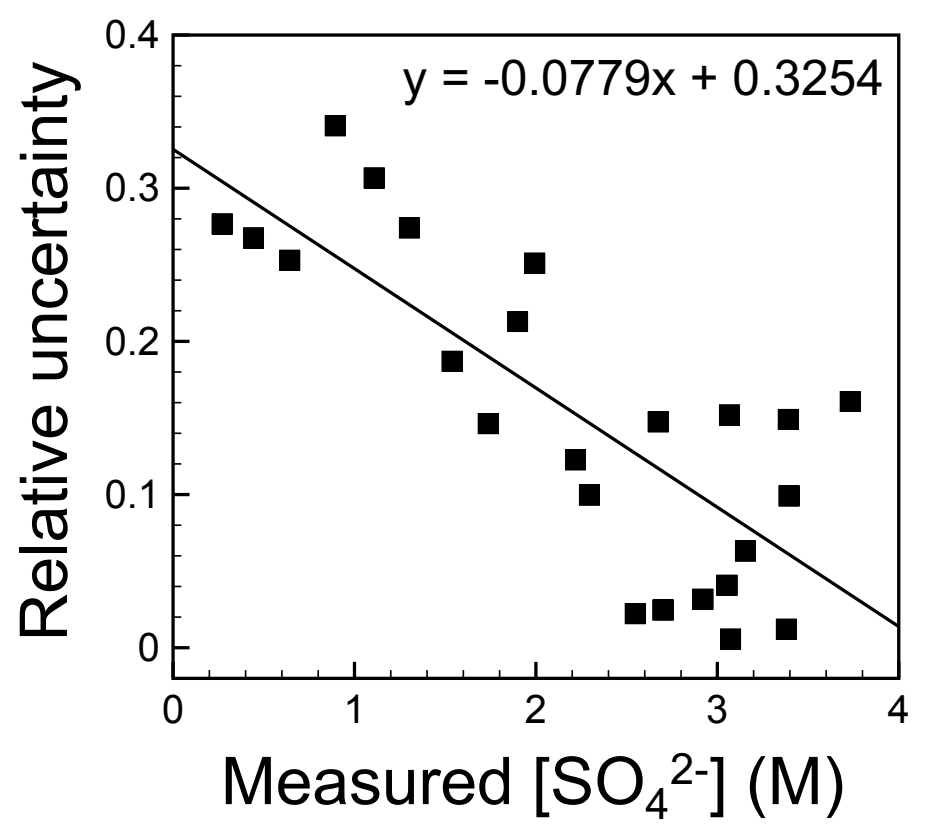

Figure S4. Plot of relative uncertainty of measured sulfate concentration, $\left[\mathrm{SO}_{4}{ }^{2-}\right]$ vs measured $\left[\mathrm{SO}_{4}{ }^{2-}\right]$. The uncertainties (one standard deviation) were calculated from the replicate experiments for irradiated particles of $\mathrm{Cl}^{-}$and $\mathrm{Fe}^{3+}$ in air (Figure S3). The solid line represents linear fit to the data. 

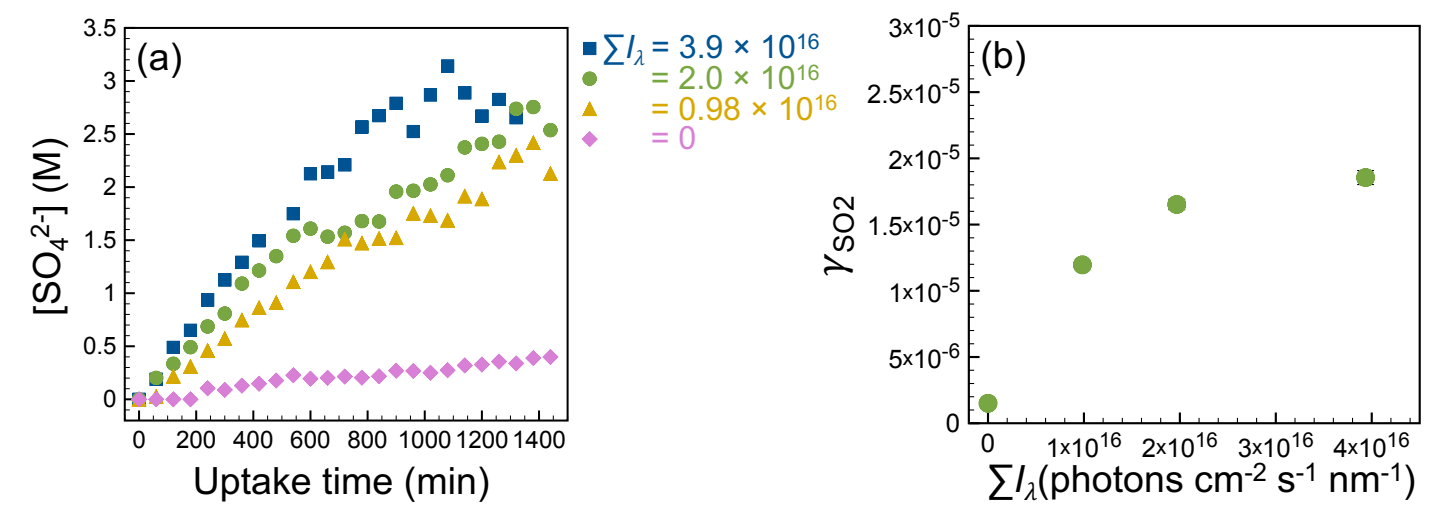

Figure S5. (a) Sulfate production for irradiated particles containing iron chlorides and (b) reactive uptake coefficients of $\mathrm{SO}_{2}$ into sulfate, $\gamma_{\mathrm{SO} 2}$, for the same particles as a function of total irradiation intensity, $\Sigma I_{\lambda}$. $\Sigma I_{\lambda}$ is obtained from the integration of the photon flux over wavelength.
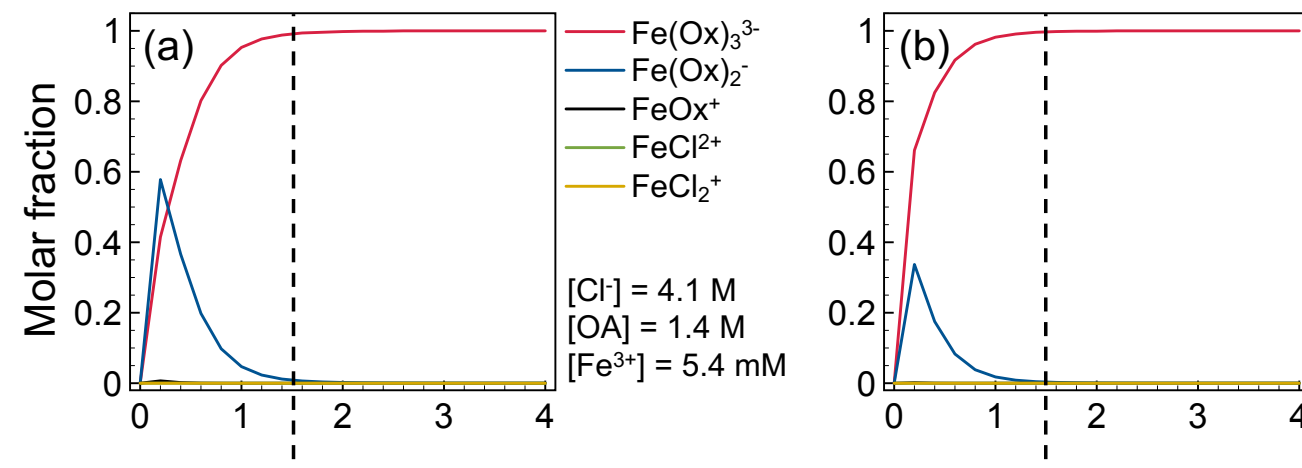

$[\mathrm{Cl}-]=3.8 \mathrm{M}$

$[\mathrm{OA}]=3.8 \mathrm{M}$ $\left[\mathrm{Fe}^{3+}\right]=7.6 \mathrm{mM}$
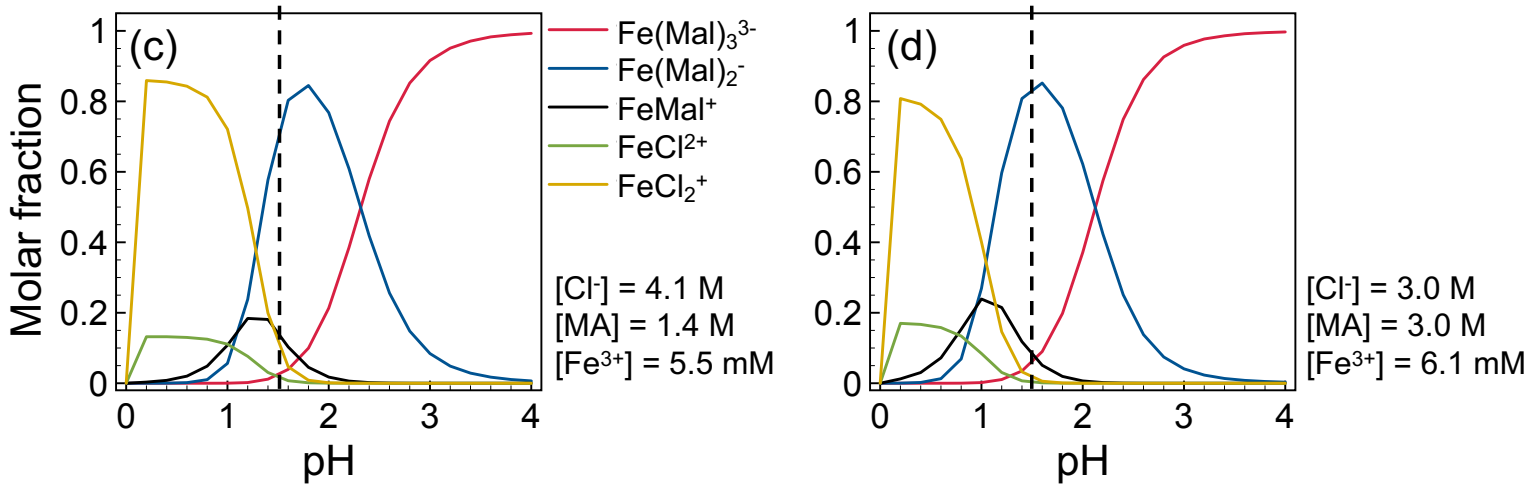

Figure S6. Distributions of iron complexes as a function of $\mathrm{pH}$ for particles of chloride, $\mathrm{Fe}^{3+}$, and $(\mathrm{a}, \mathrm{b})$ oxalic acid (OA) or (c,d) malonic acid (MA). The concentrations of chloride, $\mathrm{Fe}^{3+}$, $\mathrm{OA}$, and $\mathrm{MA},\left[\mathrm{Cl}^{-}\right],\left[\mathrm{Fe}^{3+}\right],[\mathrm{OA}]$, and $[\mathrm{MA}]$, respectively, are shown in each panel. 


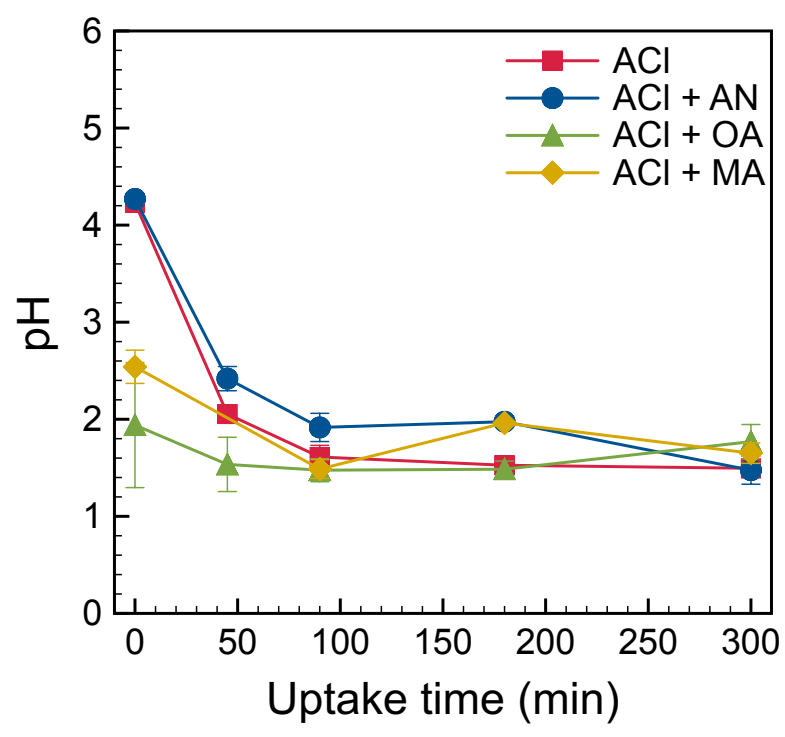

Figure S7. Variations of particle $\mathrm{pH}$ during reaction for particles of (squares) chloride $\left(\mathrm{Cl}^{-}\right)$ and $\mathrm{Fe}^{3+}$, (circles) $\mathrm{Cl}^{-}, \mathrm{Fe}^{3+}$, and nitrate, (triangles) $\mathrm{Cl}^{-}, \mathrm{Fe}^{3+}$, and oxalic acid (OA), (diamonds) $\mathrm{Cl}^{-}, \mathrm{Fe}^{3+}$, and malonic acid (MA). Following the earlier work, ${ }^{46}$ particle $\mathrm{pH}$ was measured.
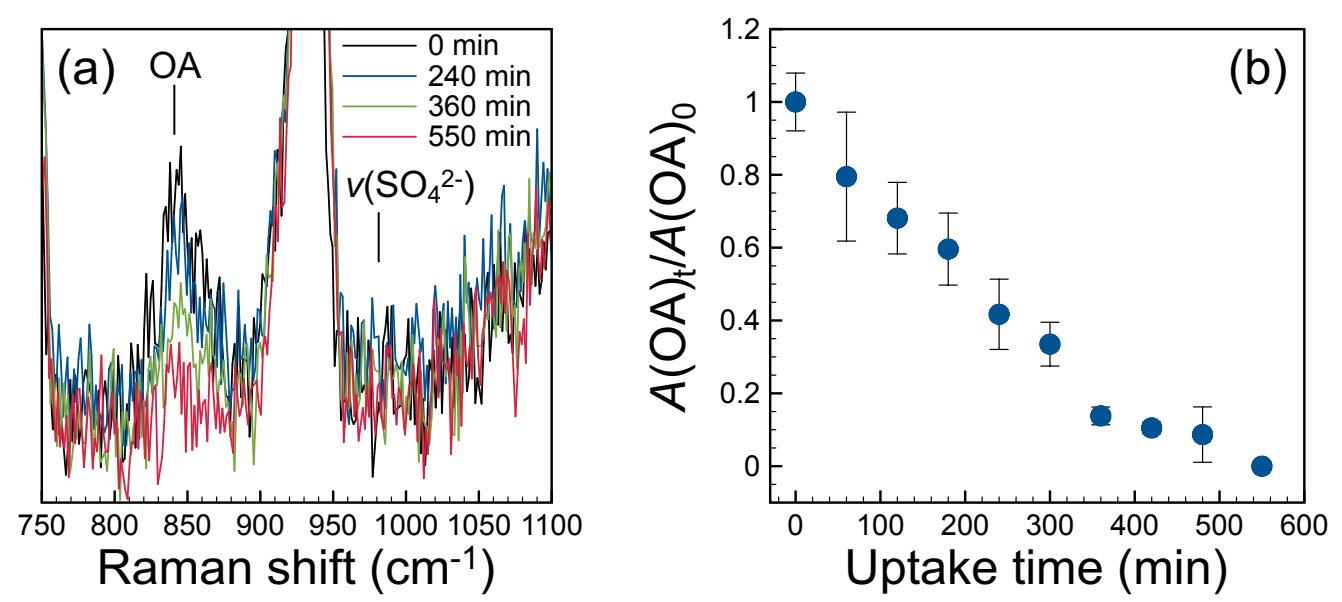

Figure S8. (a) Time-dependent Raman spectra of the particle containing $\mathrm{Fe}^{3+}$, oxalic acid (OA), and perchlorate. Raman peaks of OA and sulfate are marked. (b) Raman peak area ratio of $\mathrm{OA}$ at a given time, $A(\mathrm{OA})_{\mathrm{t}}$, and at time zero, $A(\mathrm{OA})_{0}$. The peak areas were normalized by the peak area of $v(\mathrm{O}-\mathrm{H})$, representative of the water vibration. 

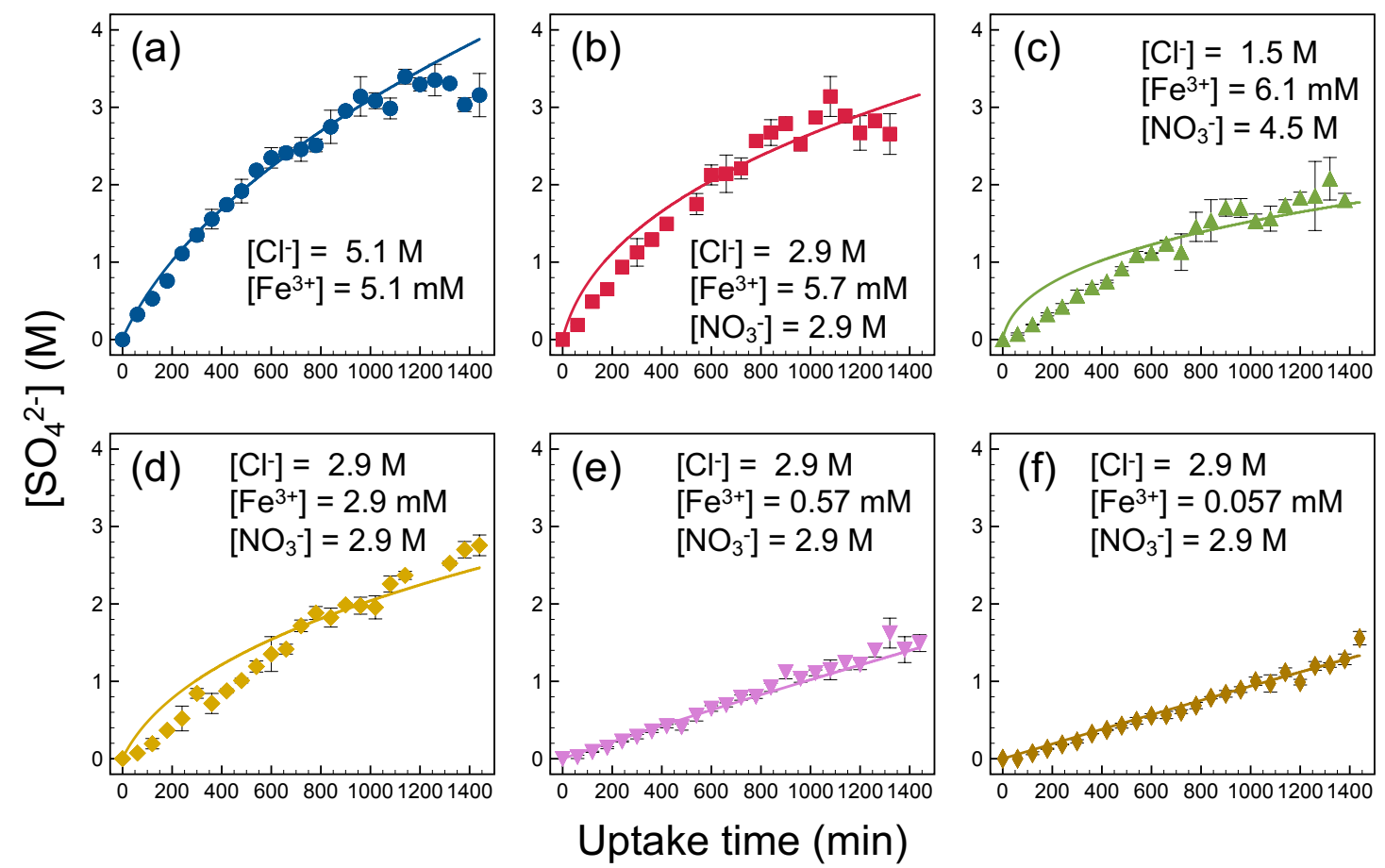

Figure S9. Model fitting to experimentally determined sulfate production for irradiated particles containing (a) chloride $\left(\mathrm{Cl}^{-}\right)$, and $\mathrm{Fe}^{3+}$, and $(\mathrm{b}-\mathrm{f}) \mathrm{Cl}^{-}, \mathrm{Fe}^{3+}$, and nitrate $\left(\mathrm{NO}_{3}{ }^{-}\right)$. The concentrations of $\mathrm{Cl}^{-}, \mathrm{Fe}^{3+}$, and $\mathrm{NO}_{3}^{-},\left[\mathrm{Cl}^{-}\right]$, $\left[\mathrm{Fe}^{3+}\right]$, and $\left[\mathrm{NO}_{3}{ }^{-}\right]$, respectively, are shown in the panels.
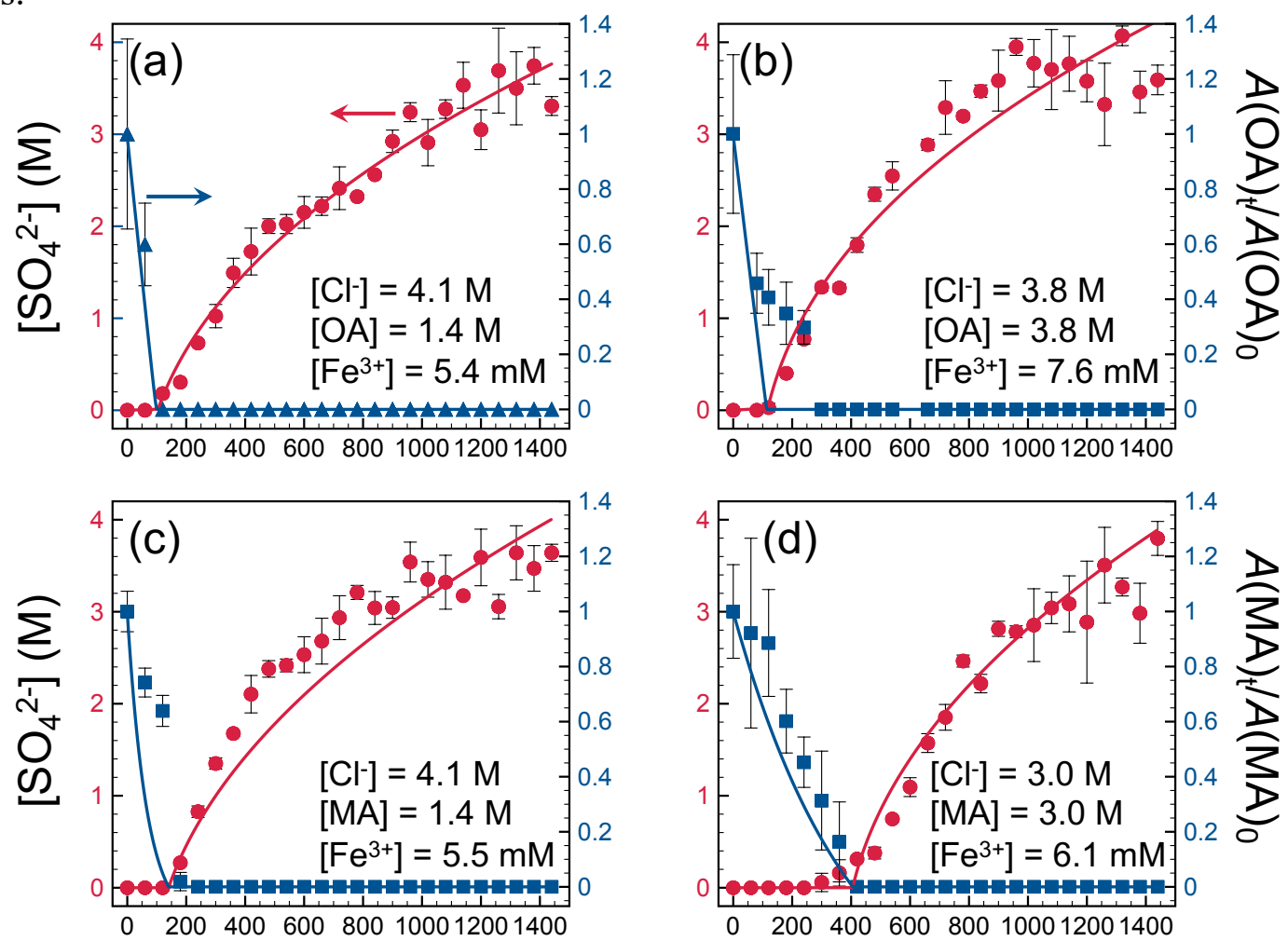

Uptake time ( $\mathrm{min})$

Figure S10. Modeling results of sulfate production for irradiated particles containing chloride, $\mathrm{Fe}^{3+}$, and $(\mathrm{a}, \mathrm{b})$ oxalic acid $(\mathrm{OA})$ or $(\mathrm{c}, \mathrm{d})$ malonic acid (MA). The concentrations of chloride, $\mathrm{Fe}^{3+}, \mathrm{OA}$, and $\mathrm{MA},\left[\mathrm{Cl}^{-}\right],\left[\mathrm{Fe}^{3+}\right]$, [OA], and [MA], respectively, are shown in each panel. 

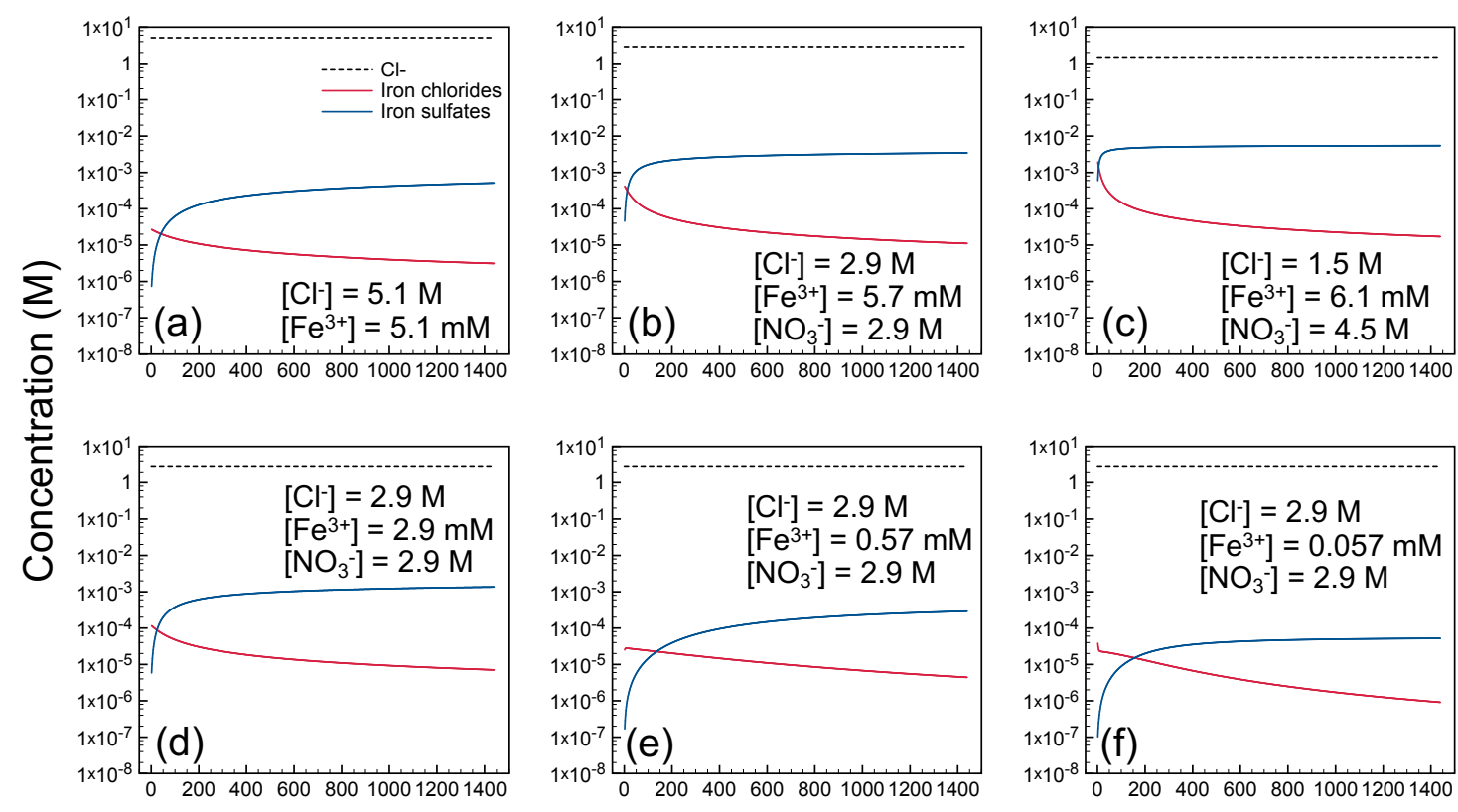

\section{Uptake time ( $\mathrm{min})$}

Figure S11. Calculated concentrations of chloride, iron chlorides $\left(\mathrm{FeCl}^{2+}\right.$ and $\left.\mathrm{FeCl}_{2}{ }^{+}\right)$, and iron sulfates $\left[\mathrm{Fe}\left(\mathrm{SO}_{4}\right)^{+}\right.$and $\left.\left.\mathrm{Fe}\left(\mathrm{SO}_{4}\right)_{2}^{-}\right)\right]$for irradiated particles containing (a) chloride $\left(\mathrm{Cl}^{-}\right)$, and $\mathrm{Fe}^{3+}$, and $(\mathrm{b}-\mathrm{f}) \mathrm{Cl}^{-}, \mathrm{Fe}^{3+}$, and nitrate $\left(\mathrm{NO}_{3}{ }^{-}\right)$. The concentrations of $\mathrm{Cl}^{-}, \mathrm{Fe}^{3+}$, and $\mathrm{NO}_{3}{ }^{-}$, $\left[\mathrm{Cl}^{-}\right],\left[\mathrm{Fe}^{3+}\right]$, and $\left[\mathrm{NO}_{3}{ }^{-}\right]$, respectively, are shown in each panel.

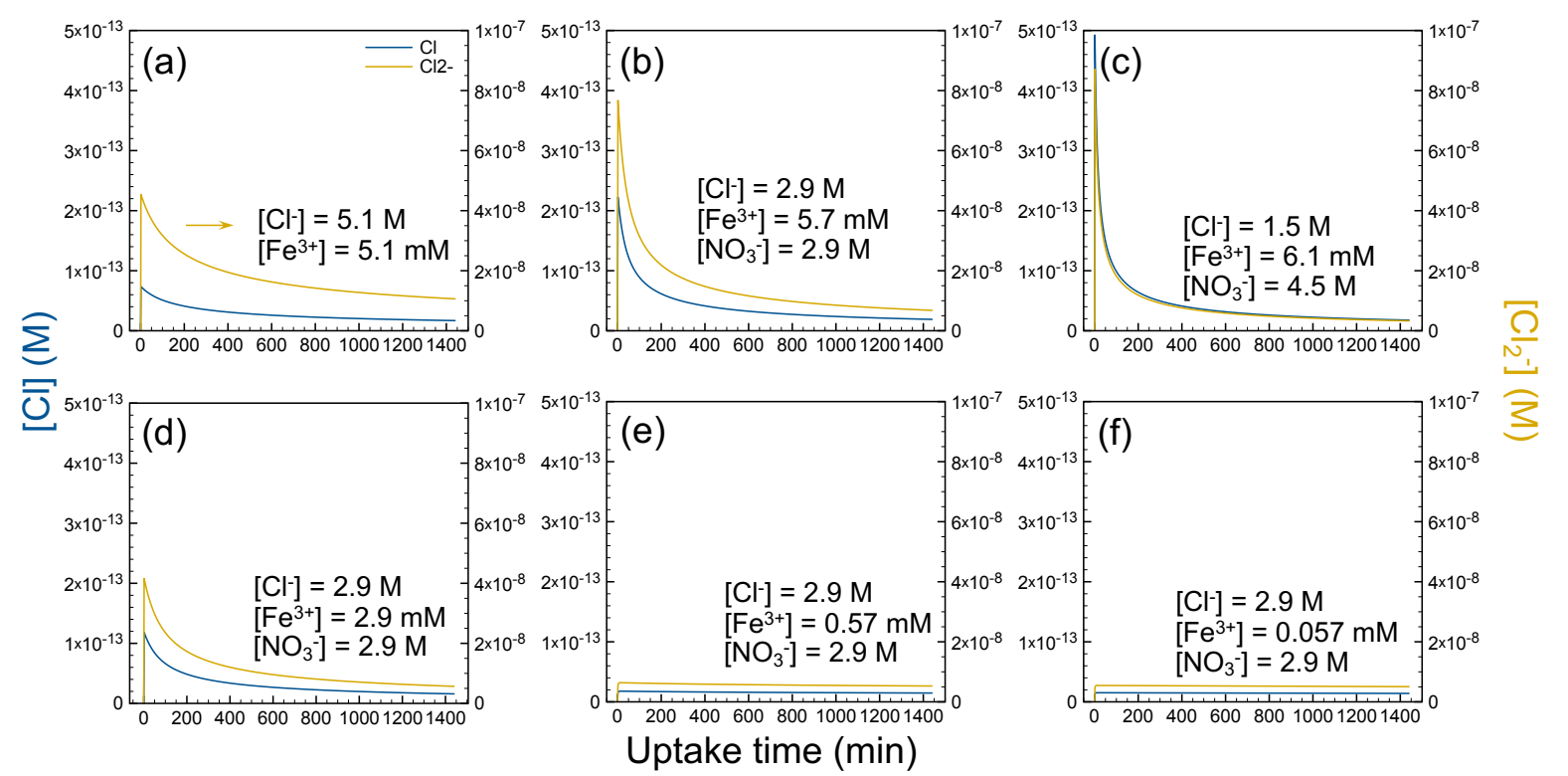

Figure S12. Calculated concentrations of $\mathrm{Cl}$ and $\mathrm{Cl}_{2}{ }^{-}$for irradiated particles containing (a) chloride $\left(\mathrm{Cl}^{-}\right)$, and $\mathrm{Fe}^{3+}$, and $(\mathrm{b}-\mathrm{f}) \mathrm{Cl}^{-}, \mathrm{Fe}^{3+}$, and nitrate $\left(\mathrm{NO}_{3}{ }^{-}\right)$. The concentrations of $\mathrm{Cl}^{-}$, $\mathrm{Fe}^{3+}$, and $\mathrm{NO}_{3}{ }^{-},\left[\mathrm{Cl}^{-}\right],\left[\mathrm{Fe}^{3+}\right]$, and $\left[\mathrm{NO}_{3}^{-}\right]$, respectively, are shown in each panel. 

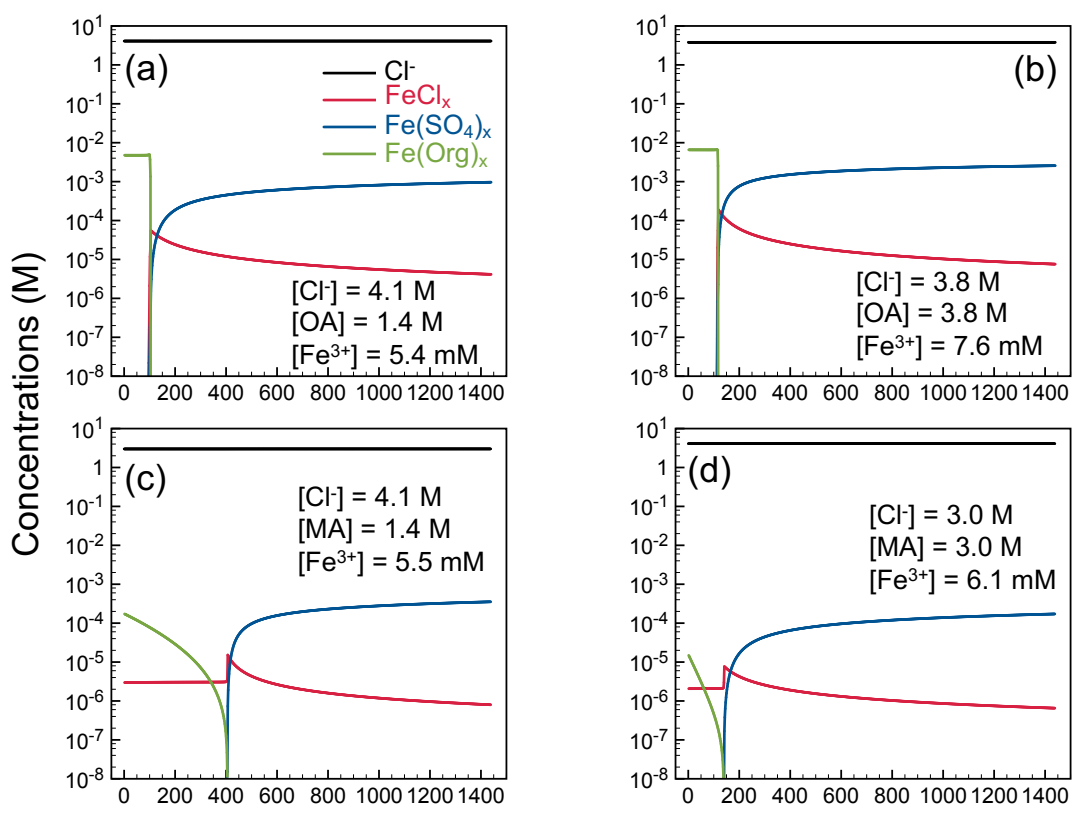

Uptake time (min)

Figure S13. Calculated concentrations of $\mathrm{Cl}^{-}$, iron(III) chlorides, $\mathrm{FeCl}_{\mathrm{x}}$, iron(III) sulfates, $\mathrm{Fe}\left(\mathrm{SO}_{4}\right)_{\mathrm{x}}$, and iron(III) organic complexes, $\mathrm{Fe}(\mathrm{Org})_{\mathrm{x}}$, for irradiated particles containing chloride, $\mathrm{Fe}^{3+}$, and $(\mathrm{a}, \mathrm{b})$ oxalic acid $(\mathrm{OA})$ or $(\mathrm{c}, \mathrm{d})$ malonic acid (MA). The concentrations of chloride, $\mathrm{Fe}^{3+}, \mathrm{OA}$, and $\mathrm{MA},\left[\mathrm{Cl}^{-}\right],\left[\mathrm{Fe}^{3+}\right]$, [OA], and [MA], respectively, are shown in each panel.
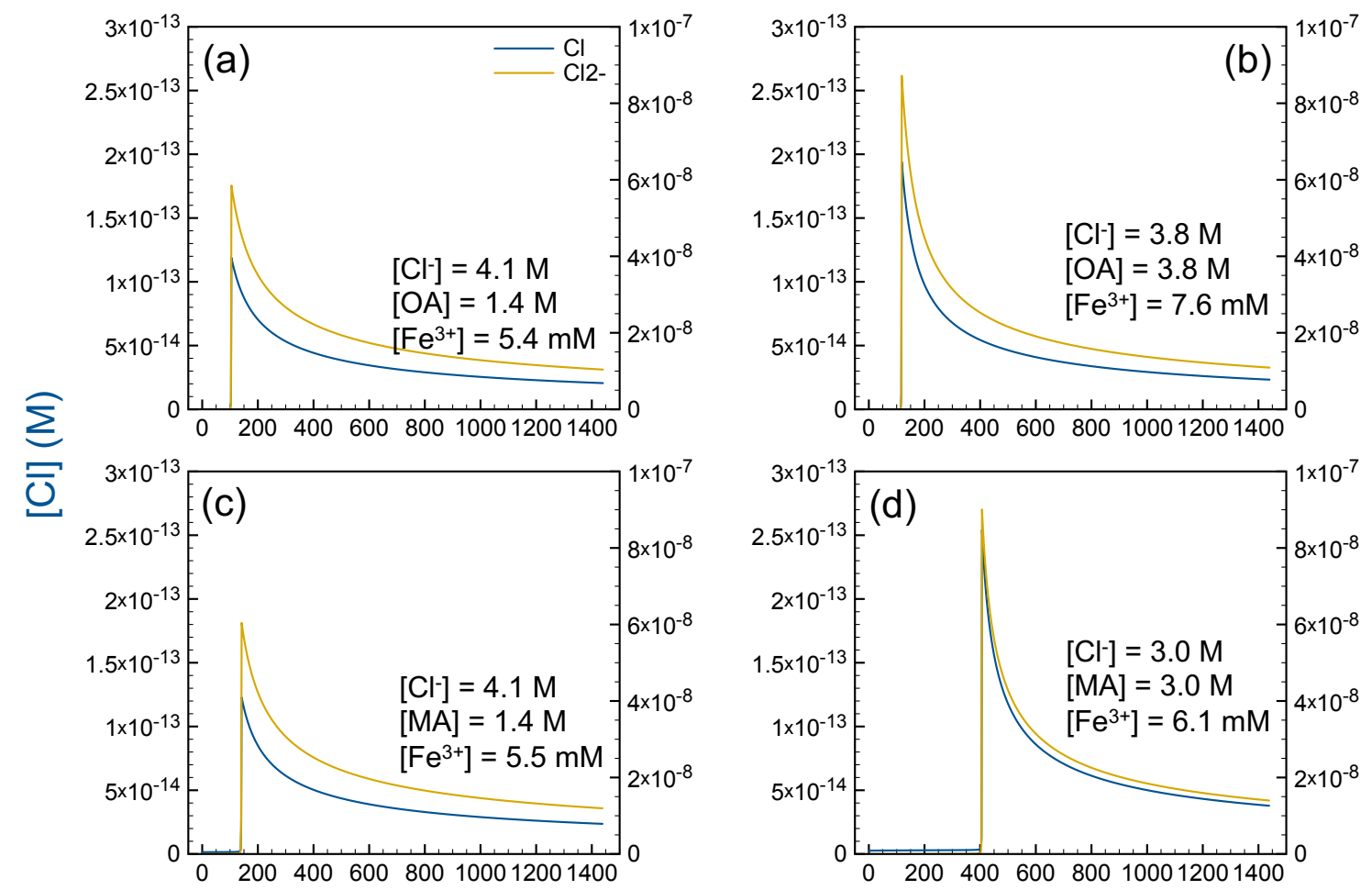

Uptake time ( $\mathrm{min})$

Figure S14. Calculated concentrations of $\mathrm{Cl}$ and $\mathrm{Cl}_{2}{ }^{-}$for irradiated particles containing chloride, $\mathrm{Fe}^{3+}$, and $(\mathrm{a}, \mathrm{b})$ oxalic acid $(\mathrm{OA})$ or $(\mathrm{c}, \mathrm{d})$ malonic acid $(\mathrm{MA})$. The concentrations of chloride, $\mathrm{Fe}^{3+}, \mathrm{OA}$, and $\mathrm{MA},\left[\mathrm{Cl}^{-}\right],\left[\mathrm{Fe}^{3+}\right],[\mathrm{OA}]$, and $[\mathrm{MA}]$, respectively, are shown in each panel. 

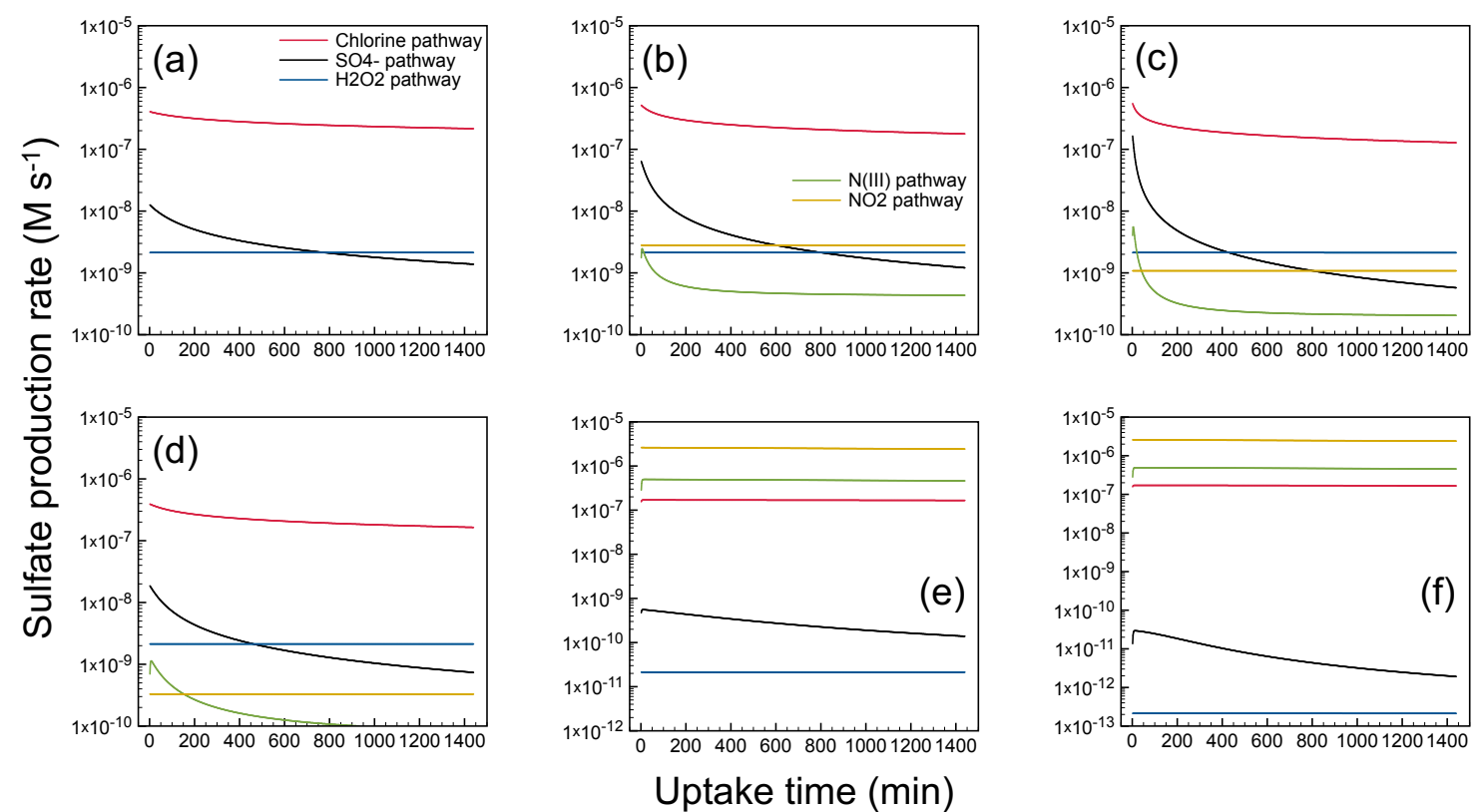

Figure S15. (a-f) Comparison of sulfate production pathways (see Text S4) for the experiments $1-6$ in Table S3, respectively.

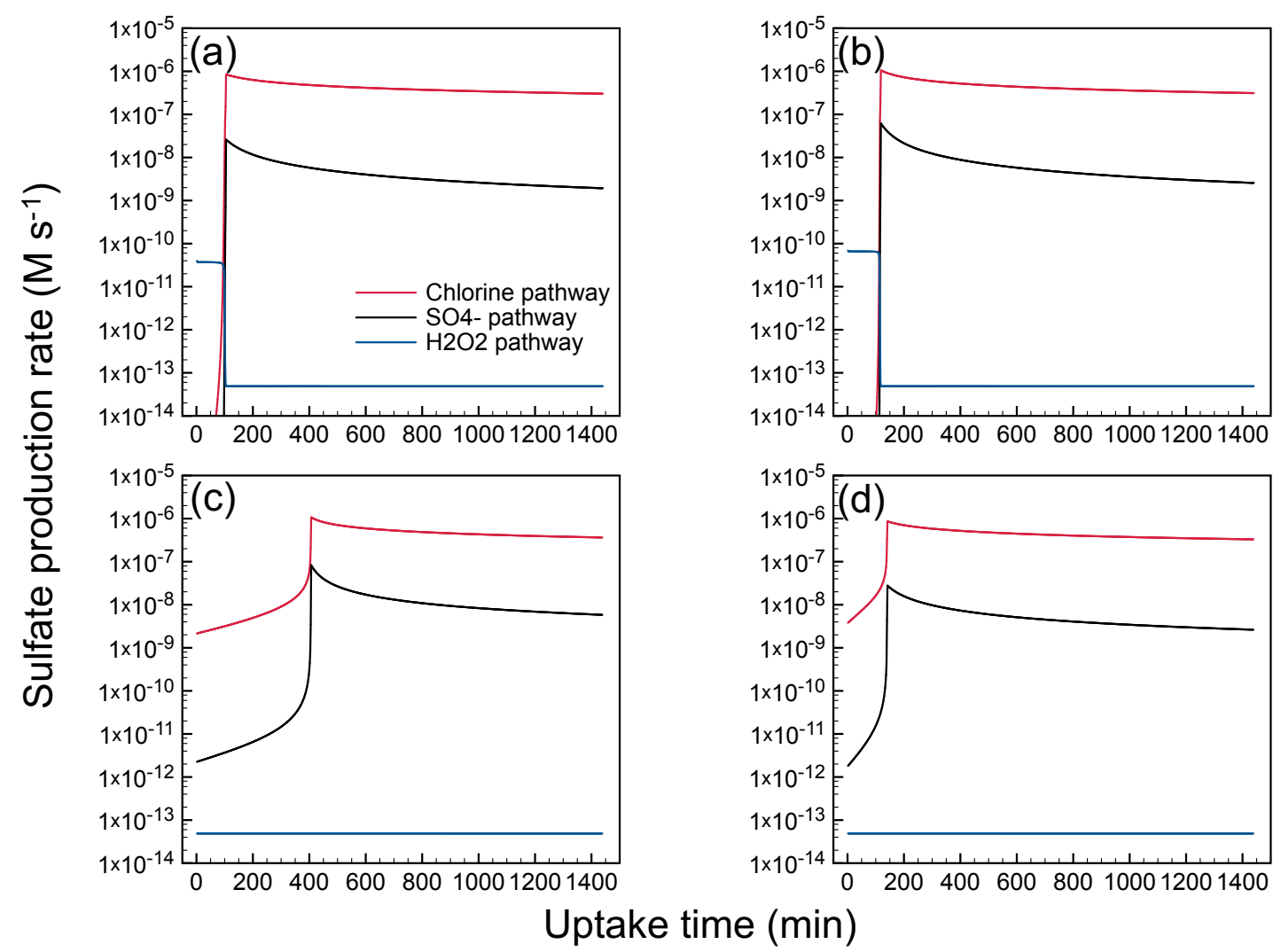

Figure S16. $(\mathrm{a}-\mathrm{d})$ Comparison of sulfate production pathways (see Text S4) for the experiments $7-10$ in Table S3, respectively. 

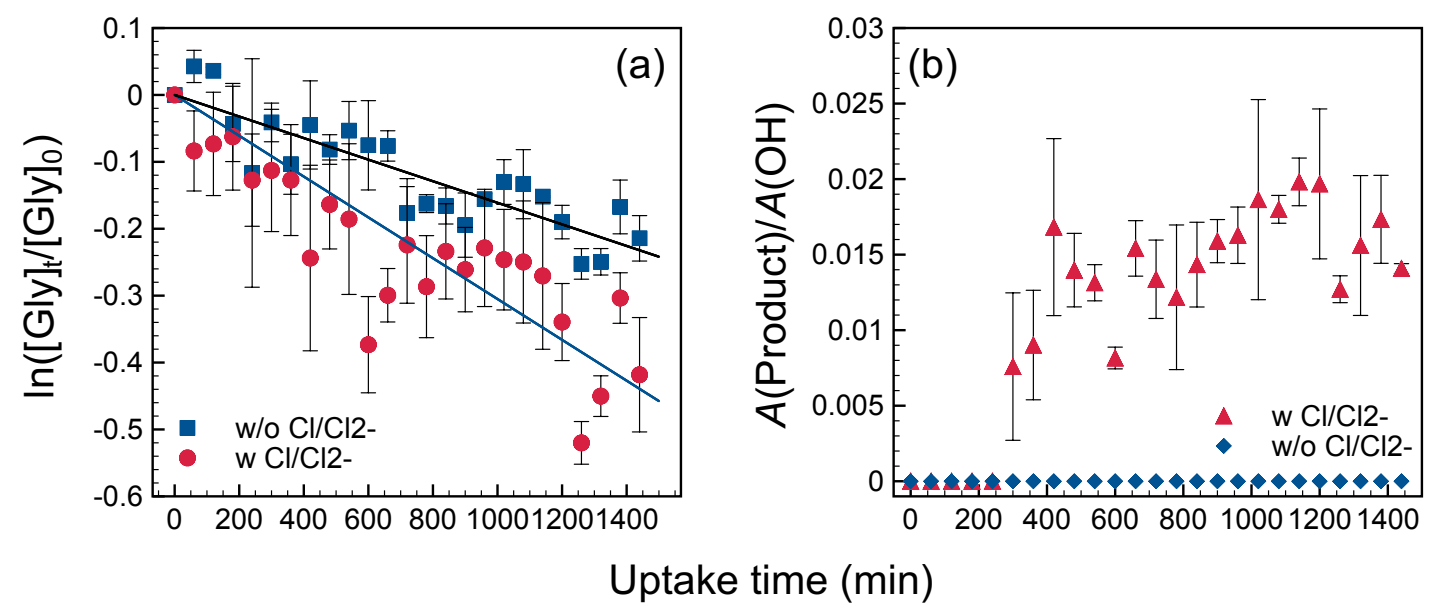

Figure S17. (a) Decay of glyoxal and (b) emergence of a peak at $1468 \mathrm{~cm}^{-1}$ as a function of uptake time during the photolysis of particles containing glyoxal, $\mathrm{Fe}^{3+}$, and chloride (w $\mathrm{Cl} / \mathrm{Cl}_{2}{ }^{-}$) or perchlorate $\left(\mathrm{w} / \mathrm{o} \mathrm{Cl} / \mathrm{Cl}_{2}{ }^{-}\right)$.

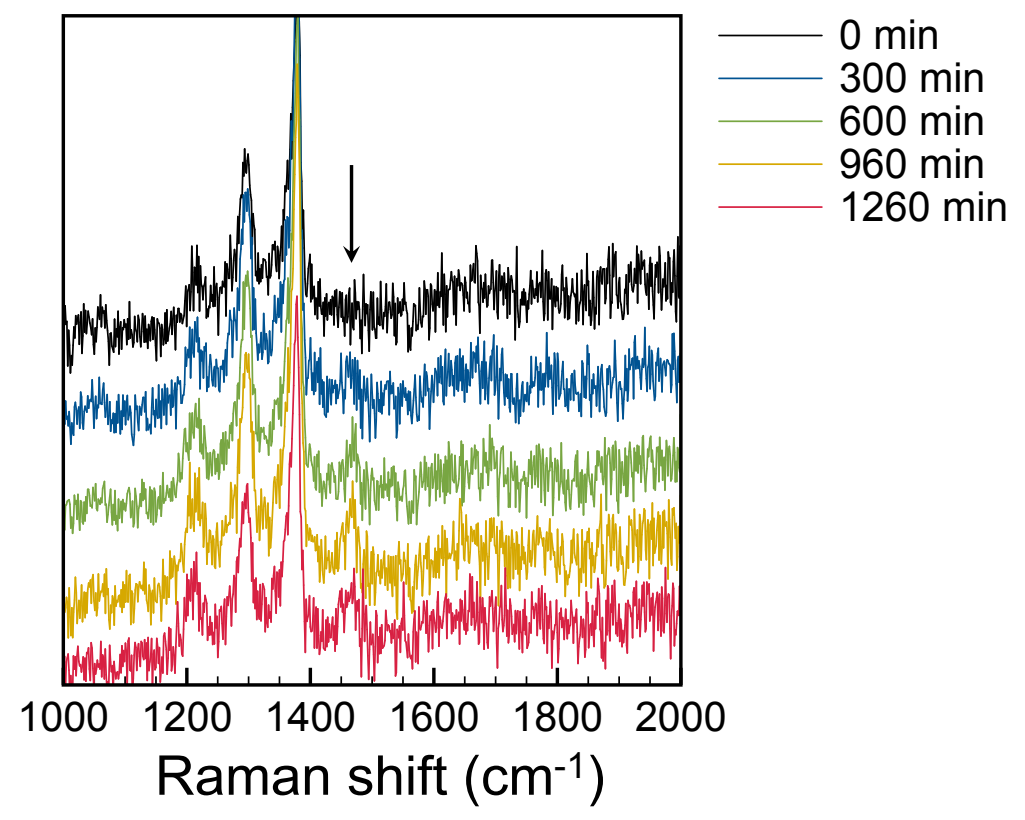

Figure S18. Raman spectra of irradiated particles containing $\mathrm{Cl}^{-}, \mathrm{Fe}^{3+}$, and glyoxal in air. The emergence of a new peak at $\sim 1470 \mathrm{~cm}^{-1}$ was observed, which can potentially be assigned to the C-O stretching mode of oxalate. ${ }^{47}$ The other peaks were due to the substrate used in this study. 


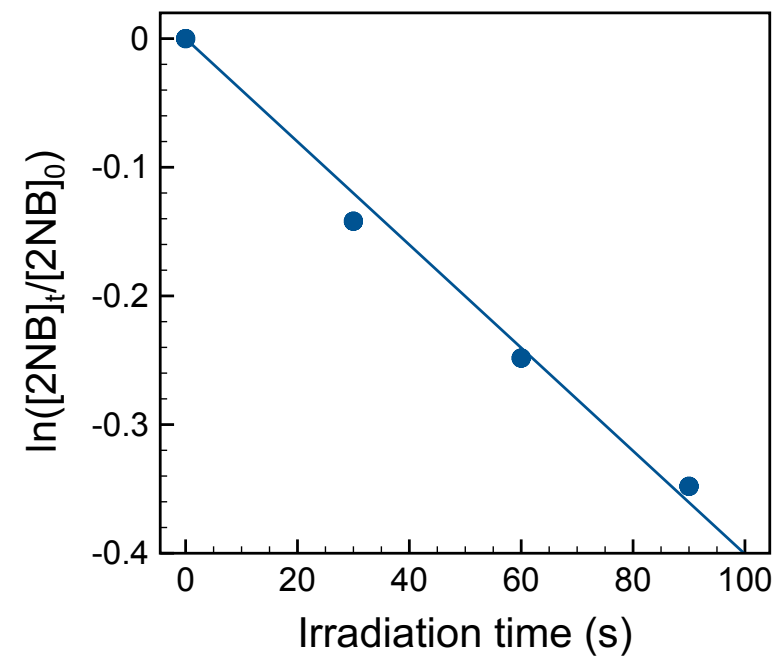

Figure S19. Photo-decay of $2 \mathrm{NB}$ as a function of irradiation time. The solid line indicates the linear regression to the $2 \mathrm{NB}$ decay, yielding the $2 \mathrm{NB}$ photo-decay rate constant.
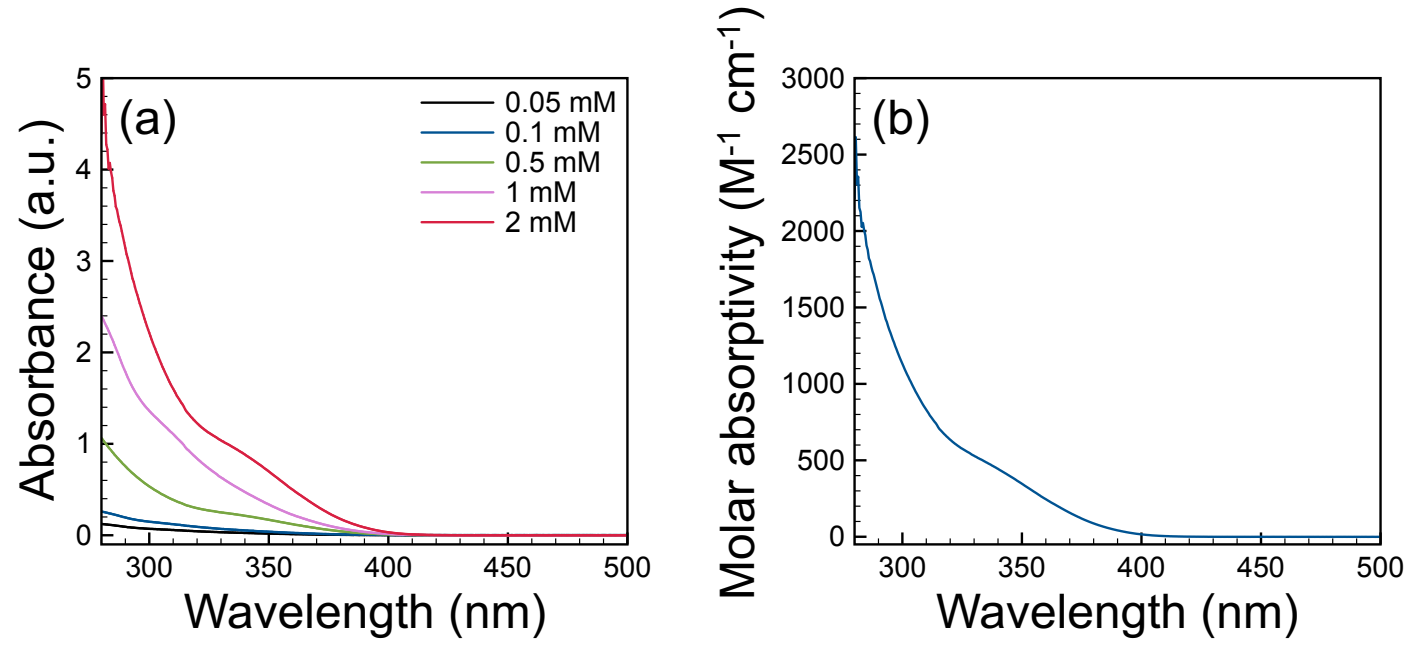

Figure S20. (a) Absorption spectra of $2 \mathrm{NB}$ in methanol as a function of $2 \mathrm{NB}$ concentration. (b) Wavelength-dependent molar absorptivity of 2NB. 


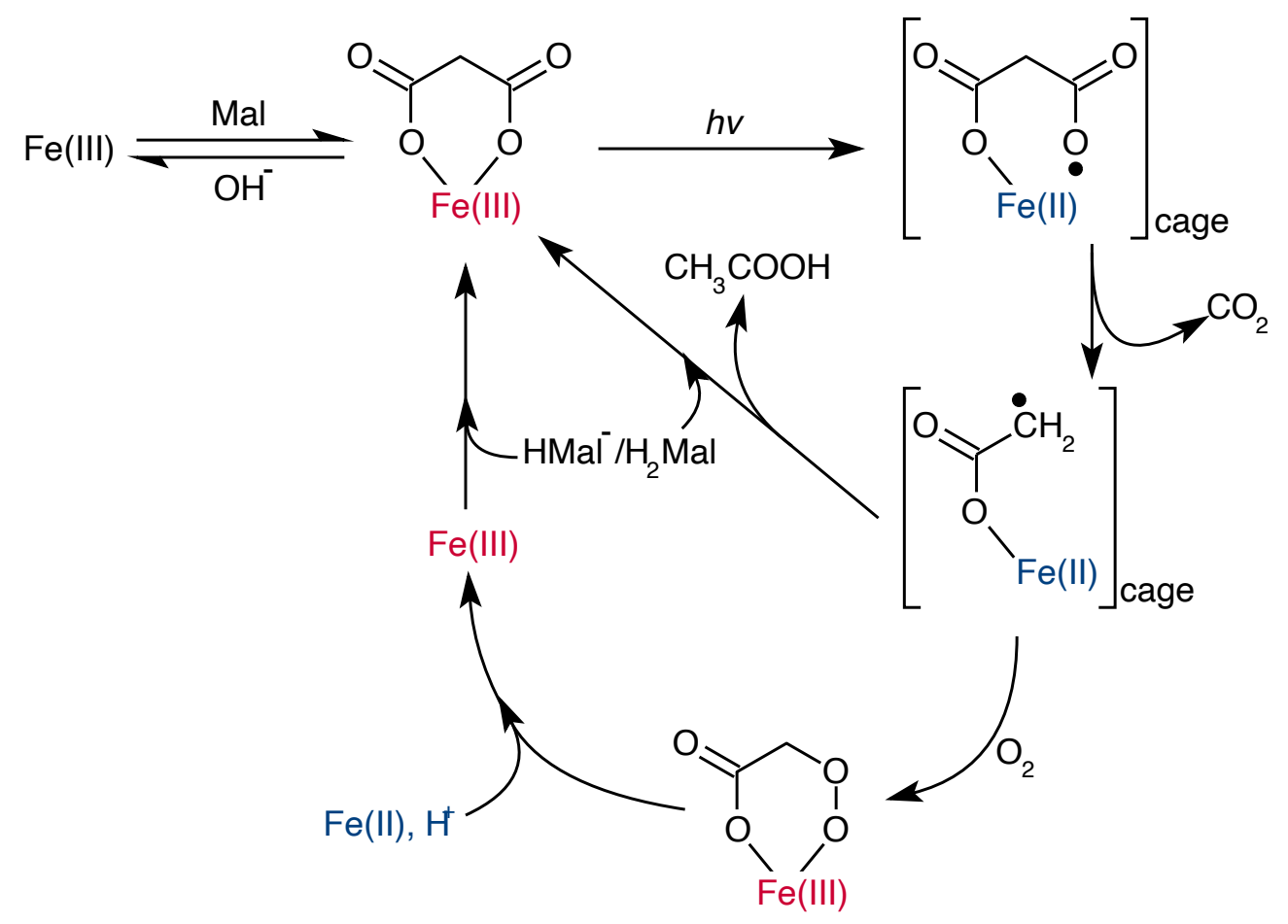

Figure S21. Reaction mechanisms involving iron and malonate (Mal). These mechanisms were adopted from the literature. ${ }^{5}$ 


\section{References}

(1) Galbavy, E. S.; Ram, K.; Anastasio, C. 2-Nitrobenzaldehyde as a Chemical Actinometer for Solution and Ice Photochemistry. J. Photochem. Photobiol. A Chem. 2010, 209 (2-3), 186-192. https://doi.org/10.1016/j.jphotochem.2009.11.013.

(2) Bianco, A.; Passananti, M.; Perroux, H.; Voyard, G.; Mouchel-Vallon, C.; Chaumerliac, N.; Mailhot, G.; Deguillaume, L.; Brigante, M. A Better Understanding of Hydroxyl Radical Photochemical Sources in Cloud Waters Collected at the Puy de Dôme Station - Experimental versus Modelled Formation Rates. Atmos. Chem. Phys. 2015, 15 (16), 9191-9202. https://doi.org/10.5194/acp-15-9191-2015.

(3) Ottley, C. J.; Davison, W.; Edmunds, W. M. Chemical Catalysis of Nitrate Reduction by Iron(II). Geochim. Cosmochim. Acta 1997, 61 (9), 1819-1828. https://doi.org/10.1016/S0016-7037(97)00058-6.

(4) Cwiertny, D. M.; Baltrusaitis, J.; Hunter, G. J.; Laskin, A.; Scherer, M. M.; Grassian, V. H. Characterization and Acid-Mobilization Study of Iron-Containing Mineral Dust Source Materials. J. Geophys. Res. Atmos. 2008, 113 (D5), D05202. https://doi.org/10.1029/2007JD009332.

(5) Wang, Z.; Chen, X.; Ji, H.; Ma, W.; Chen, C.; Zhao, J. Photochemical Cycling of Iron Mediated by Dicarboxylates: Special Effect of Malonate. Environ. Sci. Technol. 2010, 44 (1), 263-268. https://doi.org/10.1021/es901956x.

(6) Zhao, D.; Song, X.; Zhu, T.; Zhang, Z.; Liu, Y.; Shang, J. Multiphase Oxidation of $\mathrm{SO} 2$ by $\mathrm{NO} 2$ on CaCO3 Particles. Atmos. Chem. Phys. 2018, 18 (4), 2481-2493. https://doi.org/10.5194/acp-18-2481-2018.

(7) Jayson, G. G.; Parsons, B. J.; Swallow, A. J. Some Simple, Highly Reactive, Inorganic Chlorne Derivatives in Aqueous Solution. J. Chem. Soc. Faraday Trans. 1 Phys. Chem. Condens. Phases 1973, 69, 1597-1607. https://doi.org/10.1039/F19736901597.

(8) Tan, Y.; Perri, M. J.; Seitzinger, S. P.; Turpin, B. J. Effects of Precursor Concentration and Acidic Sulfate in Aqueous Glyoxal - OH Radical Oxidation and Implications for Secondary Organic Aerosol. Environ. Sci. Technol. 2009, 43 (21), 8105-8112. https://doi.org/10.1021/es901742f.

(9) Lei, Y.; Cheng, S.; Luo, N.; Yang, X.; An, T. Rate Constants and Mechanisms of the Reactions of $\mathrm{Cl} \bullet$ and $\mathrm{Cl} 2 \bullet-$ with Trace Organic Contaminants. Environ. Sci. Technol. 2019, 53 (19), 11170-11182. https://doi.org/10.1021/acs.est.9b02462.

(10) Yao, X.; Chan, C. K.; Fang, M.; Cadle, S.; Chan, T.; Mulawa, P.; He, K.; Ye, B. The Water-Soluble Ionic Composition of PM2.5 in Shanghai and Beijing, China. Atmos. Environ. 2002, 36 (26), 4223-4234. https://doi.org/10.1016/S1352-2310(02)00342-4.

(11) Yang, X.; Wang, T.; Xia, M.; Gao, X.; Li, Q.; Zhang, N.; Gao, Y.; Lee, S.; Wang, X.; Xue, L.; Yang, L.; Wang, W. Abundance and Origin of Fine Particulate Chloride in Continental China. Sci. Total Environ. 2018, 624, 1041-1051. https://doi.org/10.1016/j.scitotenv.2017.12.205.

(12) Li, T.; Wang, Y.; Li, W. J.; Chen, J. M.; Wang, T.; Wang, W. X. Concentrations and Solubility of Trace Elements in Fine Particles at a Mountain Site, Southern China: Regional Sources and Cloud Processing. Atmos. Chem. Phys. 2015, 15 (15), 89879002. https://doi.org/10.5194/acp-15-8987-2015.

(13) Cheng, Y.; Zheng, G.; Wei, C.; Mu, Q.; Zheng, B.; Wang, Z.; Gao, M.; Zhang, Q.; He, K.; Carmichael, G.; Pöschl, U.; Su, H. Reactive Nitrogen Chemistry in Aerosol Water as a Source of Sulfate during Haze Events in China. Sci. Adv. 2016, 2 (12), e1601530. https://doi.org/10.1126/sciadv.1601530.

(14) Xu, L.; Chen, X.; Chen, J.; Zhang, F.; He, C.; Zhao, J.; Yin, L. Seasonal Variations and Chemical Compositions of PM 2.5 Aerosol in the Urban Area of Fuzhou, China. Atmos. Res. 2012, 104-105, 264-272. https://doi.org/10.1016/j.atmosres.2011.10.017. 
(15) Ye, B.; Ji, X.; Yang, H.; Yao, X.; Chan, C. K.; Cadle, S. H.; Chan, T.; Mulawa, P. A. Concentration and Chemical Composition of PM2.5 in Shanghai for a 1-Year Period. Atmos. Environ. 2003, 37 (4), 499-510. https://doi.org/10.1016/S13522310(02)00918-4.

(16) Yin, L.; Niu, Z.; Chen, X.; Chen, J.; Xu, L.; Zhang, F. Chemical Compositions of PM2.5 Aerosol during Haze Periods in the Mountainous City of Yong'an, China. $J$. Environ. Sci. (China) 2012, 24 (7), 1225-1233. https://doi.org/10.1016/S10010742(11)60940-6.

(17) Tao, J.; Zhang, L.; Engling, G.; Zhang, R.; Yang, Y.; Cao, J.; Zhu, C.; Wang, Q.; Luo, L. Chemical Composition of PM2.5 in an Urban Environment in Chengdu, China: Importance of Springtime Dust Storms and Biomass Burning. Atmos. Res. 2013, 122, 270-283. https://doi.org/10.1016/j.atmosres.2012.11.004.

(18) Yang, L.; Zhou, X.; Wang, Z.; Zhou, Y.; Cheng, S.; Xu, P.; Gao, X.; Nie, W.; Wang, X.; Wang, W. Airborne Fine Particulate Pollution in Jinan, China: Concentrations, Chemical Compositions and Influence on Visibility Impairment. Atmos. Environ. 2012, 55, 506-514. https://doi.org/10.1016/j.atmosenv.2012.02.029.

(19) Yang, F.; Ye, B.; He, K.; Ma, Y.; Cadle, S. H.; Chan, T.; Mulawa, P. A. Characterization of Atmospheric Mineral Components of PM2.5 in Beijing and Shanghai, China. Sci. Total Environ. 2005, 343 (1-3), 221-230. https://doi.org/10.1016/j.scitotenv.2004.10.017.

(20) Geng, N.; Wang, J.; Xu, Y.; Zhang, W.; Chen, C.; Zhang, R. PM2.5 in an Industrial District of Zhengzhou, China: Chemical Composition and Source Apportionment. Particuology 2013, 11 (1), 99-109. https://doi.org/10.1016/j.partic.2012.08.004.

(21) Yao, X.; Fang, M.; Chan, C. K. Size Distributions and Formation of Dicarboxylic Acids in Atmospheric Particles. Atmos. Environ. 2002, 36 (13), 2099-2107. https://doi.org/10.1016/S1352-2310(02)00230-3.

(22) Weller, C.; Tilgner, A.; Bräuer, P.; Herrmann, H. Modeling the Impact of IronCarboxylate Photochemistry on Radical Budget and Carboxylate Degradation in Cloud Droplets and Particles. Environ. Sci. Technol. 2014, 48 (10), 5652-5659. https://doi.org/10.1021/es4056643.

(23) Baker, A. R.; Jickells, T. D.; Witt, M.; Linge, K. L. Trends in the Solubility of Iron, Aluminium, Manganese and Phosphorus in Aerosol Collected over the Atlantic Ocean. Mar. Chem. 2006, 98 (1), 43-58. https://doi.org/10.1016/j.marchem.2005.06.004.

(24) Sedwick, P. N.; Sholkovitz, E. R.; Church, T. M. Impact of Anthropogenic Combustion Emissions on the Fractional Solubility of Aerosol Iron: Evidence from the Sargasso Sea. Geochemistry, Geophys. Geosystems 2007, 8 (10), Q10Q06. https://doi.org/10.1029/2007GC001586.

(25) Siefert, R. L.; Johansen, A. M.; Hoffmann, M. R.; Pehkonen, S. O. Measurements of Trace Metal (Fe, $\mathrm{Cu}, \mathrm{Mn}, \mathrm{Cr})$ Oxidation States in Fog and Stratus Clouds. J. Air Waste Manag. Assoc. 1998, 48 (2), 128-143. https://doi.org/10.1080/10473289.1998.10463659.

(26) Nadtochenko, V. A.; Kiwi, J. Photolysis of FeOH2+ and FeCl2+ in Aqueous Solution. Photodissociation Kinetics and Quantum Yields. Inorg. Chem. 1998, 37 (20), 52335238. https://doi.org/10.1021/ic9804723.

(27) Kiwi, J.; Lopez, A.; Nadtochenko, V. Mechanism and Kinetics of the OH-Radical Intervention during Fenton Oxidation in the Presence of a Significant Amount of Radical Scavenger (Cl-). Environ. Sci. Technol. 2000, 34 (11), 2162-2168. https://doi.org/10.1021/es991406i.

(28) Benkelberg, H. J.; Warneck, P. Photodecomposition of Iron(III) Hydroxo and Sulfato Complexes in Aqueous Solution: Wavelength Dependence of OH and 804- Quantum 
Yields. J. Phys. Chem. 1995, 99 (14), 5214-5221.

https://doi.org/10.1021/j100014a049.

(29) De Laat, J.; Le, T. G. Effects of Chloride Ions on the Iron(III)-Catalyzed

Decomposition of Hydrogen Peroxide and on the Efficiency of the Fenton-like

Oxidation Process. Appl. Catal. B Environ. 2006, 66 (1), 137-146.

https://doi.org/https://doi.org/10.1016/j.apcatb.2006.03.008.

(30) Huie, R. E.; Neta, P. Rate Constants for Some Oxidations of S(IV) by Radicals in Aqueous Solutions. Atmos. Environ. 1987, 21 (8), 1743-1747.

https://doi.org/10.1016/0004-6981(87)90113-2.

(31) Machulek Jr., A.; Moraes, J. E. F.; Okano, L. T.; Silvério, C. A.; Quina, F. H.

Photolysis of Ferric Ions in the Presence of Sulfate or Chloride Ions: Implications for the Photo-Fenton Process. Photochem. Photobiol. Sci. 2009, 8 (7), 985-991. https://doi.org/10.1039/B900553F.

(32) Seinfeld, J. H.; Pandis, S. N. Atmospheric Chemistry and Physics: From Air Pollution to Climate Change; Wiley: New York, 2006. https://doi.org/10.1063/1.882420.

(33) Bal Reddy, K.; van Eldik, R. Kinetics and Mechanism of the Sulfite-Induced Autoxidation of Fe(II) in Acidic Aqueous Solution. Atmos. Environ. Part A, Gen. Top. 1992, 26 (4), 661-665. https://doi.org/10.1016/0960-1686(92)90177-M.

(34) Ziajka, J.; Beer, F.; Warneck, P. Iron-Catalysed Oxidation of Bisulphite Aqueous Solution: Evidence for a Free Radical Chain Mechanism. Atmos. Environ. 1994, 28 (15), 2549-2552. https://doi.org/10.1016/1352-2310(94)90405-7.

(35) Mack, J.; Bolton, J. R. Photochemistry of Nitrite and Nitrate in Aqueous Solution: A Review. J. Photochem. Photobiol. A Chem. 1999, 128 (1-3), 1-13. https://doi.org/10.1016/S1010-6030(99)00155-0.

(36) Oblath, S. B.; Markowitz, S. S.; Novakov, T.; Chang, S. G. Kinetics of the Formation of Hydroxylamine Disulfonate by Reaction of Nitrite with Sulfites. J. Phys. Chem. 1981, 85 (8), 1017-1021. https://doi.org/10.1021/j150608a018.

(37) Oblath, S. B.; Markowitz, S. S.; Novakov, T.; Chang, S. G. Kinetics of the Initial Reaction of Nitrite Ion in Bisulfite Solutions. J. Phys. Chem. 1982, 86 (25), 48534857. https://doi.org/10.1021/j100222a005.

(38) Cruywagen, J. J.; Heyns, J. B. B. Determination of the Dissociation Constants of Oxalic Acid and the Ultraviolet Spectra of the Oxalate Species in 3M Perchlorate Medium. Talanta 1983, 30 (3), 197-200. https://doi.org/10.1016/00399140(83)80051-4.

(39) Balmer, M. E.; Sulzberger, B. Atrazine Degradation in Irradiated Iron/Oxalate Systems: Effects of PH and Oxalate. Environ. Sci. Technol. 1999, 33 (14), 2418-2424. https://doi.org/10.1021/es9808705.

(40) Weller, C.; Horn, S.; Herrmann, H. Effects of Fe(III)-Concentration, Speciation, Excitation-Wavelength and Light Intensity on the Quantum Yield of Iron(III)-Oxalato Complex Photolysis. J. Photochem. Photobiol. A Chem. 2013, 255, 41-49. https://doi.org/https://doi.org/10.1016/j.jphotochem.2013.01.014.

(41) Wicktor, F.; Donati, A.; Herrmann, H.; Zellner, R. Laser Based Spectroscopic and Kinetic Investigations of Reactions of the $\mathrm{Cl}$ Atom with Oxygenated Hydrocarbons in Aqueous Solution. Phys. Chem. Chem. Phys. 2003, 5 (12), 2562-2572. https://doi.org/10.1039/b212666d.

(42) Burton, J. O.; Hamer, W. J.; Acree, S. F. Dissociation Constants of Malonic Acid in Its Sodium-Salt Solutions at 25 Degrees C from Electrometric Titration Measurements. $J$. Res. Natl. Bur. Stand. (1934). 1936, 16, 575-593. https://doi.org/10.6028/jres.016.037.

(43) Salvadó, V.; Ribas, X.; Zelano, V.; Ostacoli, G.; Valiente, M. The Chemistry of Iron in Biosystems - III. Complex Formation between FeIII and Malonic Acid in Aqueous 
Solutions. Polyhedron 1989, 8 (6), 813-818.

https://doi.org/https://doi.org/10.1016/S0277-5387(00)83851-6.

(44) Chu, L.; Anastasio, C. Quantum Yields of Hydroxyl Radical and Nitrogen Dioxide from the Photolysis of Nitrate on Ice. J. Phys. Chem. A 2003.

https://doi.org/10.1021/jp0349132.

(45) Byrne, R. H.; Kester, D. R. Ultraviolet Spectroscopic Study of Ferric Equilibria at High Chloride Concentrations. J. Solution Chem. 1981, 10 (1), 51-67.

https://doi.org/10.1007/BF00652780.

(46) Craig, R. L.; Peterson, P. K.; Nandy, L.; Lei, Z.; Hossain, M. A.; Camarena, S.; Dodson, R. A.; Cook, R. D.; Dutcher, C. S.; Ault, A. P. Direct Determination of Aerosol PH: Size-Resolved Measurements of Submicrometer and Supermicrometer Aqueous Particles. Anal. Chem. 2018, 90 (19), 11232-11239. https://doi.org/10.1021/acs.analchem.8b00586.

(47) Frost, R. L.; Weier, M. L. Raman Spectroscopy of Natural Oxalates at 298 and 77 K. J. Raman Spectrosc. 2003, 34 (10), 776-785. https://doi.org/10.1002/jrs.1052. 